\title{
The HYTHIRM Project: Flight Thermography of the Space Shuttle During Hypersonic Re-entry
}

Thomas J. Horvath, Deborah M. Tomek, Karen T. Berger, Scott C. Splinter, Joseph N. Zalameda and Paul W. Krasa NASA Langley Research Center, Hampton VA 23681

\author{
Steve Tack \\ Naval Air Warfare Center - Weapons Division, Pt. Mugu, CA 93042 \\ Richard J. Schwartz \\ ATK, Hampton, VA 23681 \\ David M. Gibson \\ Johns Hopkins University Applied Physics Laboratory, Laurel, MD 20723 \\ Alan Tietjen \\ ISTEF-CSC, Kennedy Space Center, FL 32899
}

\begin{abstract}
This report describes a NASA Langley led endeavor sponsored by the NASA Engineering Safety Center, the Space Shuttle Program Office and the NASA Aeronautics Research Mission Directorate to demonstrate a quantitative thermal imaging capability. A background and an overview of several multidisciplinary efforts that culminated in the acquisition of high resolution calibrated infrared imagery of the Space Shuttle during hypervelocity atmospheric entry is presented. The successful collection of thermal data has demonstrated the feasibility of obtaining remote high-resolution infrared imagery during hypersonic flight for the accurate measurement of surface temperature. To maximize science and engineering return, the acquisition of quantitative thermal imagery and capability demonstration was targeted towards three recent Shuttle flights - two of which involved flight experiments flown on Discovery. In coordination with these two Shuttle flight experiments, a US Navy NP-3D aircraft was flown between 26-41 nautical miles below Discovery and remotely monitored surface temperature of the Orbiter at Mach 8.4 (STS-119) and Mach 14.7 (STS-128) using a long-range infrared optical package referred to as Cast Glance. This same Navy aircraft successfully monitored the Orbiter Atlantis traveling at approximately Mach $\mathbf{1 4 . 3}$ during its return from the successful Hubble repair mission (STS-125). The purpose of this paper is to describe the systematic approach used by the Hypersonic Thermodynamic Infrared Measurements team to develop and implement a set of mission planning tools designed to establish confidence in the ability of an imaging platform to reliably acquire, track and return global quantitative surface temperatures of the Shuttle during entry. The mission planning tools included a pre-flight capability to predict the infrared signature of the Shuttle. Such tools permitted optimization of the hardware configuration to increase signal-to-noise and to maximize the available dynamic range while mitigating the potential for saturation. Post flight, analysis tools were used to assess atmospheric effects and to convert the 2-D intensity images to 3-D temperature maps of the windward surface. Comparison of the spatially resolved global thermal measurements to surface thermocouples and CFD prediction is made. Successful demonstration of a quantitative, spatially resolved, global temperature measurement on the Shuttle suggests future applications towards hypersonic flight test programs within NASA, DoD and DARPA along with flight test opportunities supporting NASA's Constellation program.
\end{abstract}

\section{Nomenclature}

$\begin{array}{ll}M & \text { freestream Mach number } \\ \mathrm{nm} & \text { nautical miles } \\ T & \text { surface temperature, deg F } \\ \alpha & \text { angle of attack, deg }\end{array}$

\section{Acronyms}

ARMD NASA Aeronautics Research Mission Directorate

BLT boundary layer transition

CFD computational fluid dynamics

DoD Department of Defense

DARPA Defense Advanced Research Project Agency 


$\begin{array}{ll}\text { HYTHIRM } & \text { Hypersonic Thermodynamic Infrared Measurements } \\ \text { IR } & \text { infrared } \\ \text { ISS } & \text { International Space Station } \\ \text { MWIR } & \text { mid-wave infrared } \\ \text { NESC } & \text { NASA Engineering and Safety Center } \\ \text { NIR } & \text { near infrared } \\ \text { NSTTF } & \text { National Solar Thermal Test Facility } \\ \text { PCA } & \text { point of closest approach } \\ \text { RCG } & \text { reaction cured glass } \\ \text { RCS } & \text { reaction control system } \\ \text { RTF } & \text { Return to Flight } \\ \text { SSP } & \text { Space Shuttle Program } \\ \text { SWIR } & \text { short-wave infrared } \\ \text { STS } & \text { Space Transportation System } \\ \text { TPS } & \text { thermal protection system } \\ \text { ViDI } & \text { Virtual Diagnostics Interface }\end{array}$

\section{Introduction}

Quantitative thermal imagery obtained by the Hypersonic Thermodynamic Infrared Measurements (HYTHIRM) team provided a unique and never before observed perspective on the global distribution of surface temperature and the state of the boundary layer (i.e., laminar/turbulent) over the entire windward surface of the Shuttle during portions of hypersonic re-entry. The quantitative thermal imagery represented several years of advocacy within the aerothermodynamics technical community, sponsorship by the NASA Engineering Safety Center, the Space Shuttle Program Office and the Hypersonics project within the NASA Aeronautics Research Mission Directorate. The partnership resulted in methodical planning and mission execution by a coalition of NASA, Navy, government labs, and contractor personnel. On Space Shuttle Discovery's 2009 STS-119 mission, NASA flew a specially modified thermal protection system tile and instrumentation package to monitor heating effects from boundary layer transition during re-entry ${ }^{1}$ (boundary layer transition occurs when the smooth, laminar flow of air close to a vehicle' surface is disturbed and becomes turbulent - resulting in surface temperature increases). On STS-119, the windward airflow on the port wing was deliberately disrupted by a four-inch wide and quarter-inch tall "speed bump" built into the modified tile - intended to promote transition to turbulence near Mach 15. In coordination with this flight experiment, the HYTHIRM team positioned a US Navy NP-3D Orion aircraft approximately 26 nautical miles below Discovery and remotely monitored surface temperature of the Orbiter at Mach 8.4 using a long-range infrared optical package referred to as Cast Glance. The imagery from this mission not only captured the expected thermal footprint of the turbulent flow downstream of the wing protuberance but a much larger area of turbulent flow on the opposing wing that was not anticipated. The global thermal imagery obtained from the aircraft complemented the data collected with an onboard instrumentation package consisting of 10 surface thermocouples.

Approximately two months later, the same Navy aircraft successfully monitored the surface temperatures of the Orbiter Atlantis (not configured with a speed bump) traveling at Mach 14.3 during its return from the successful Hubble repair mission. Six months after the first flight experiment, Discovery outfitted with a slightly taller bump intended to promote transition to turbulence near Mach 18, was successfully imaged by the P-3 at Mach 14.7. To the author's knowledge, the thermal imagery captured by the HYTHIRM team represents the first time the entire surface temperature distribution of a hypersonic vehicle has been captured in flight at these Mach numbers. Collectively, the spatially resolved global thermal measurements made by HYTHIRM during the Shuttle's hypersonic reentry were intended to demonstrate the capability to collect scientific quality imagery in a reliable manner using available technology. It is the intent of the project to analyze the imagery and provide the technical community with another source of flight data for reducing the uncertainty associated with present day ground-to-flight extrapolation techniques and current state-of-the-art empirical boundary-layer transition or turbulent heating prediction methods. Laminar and turbulent flight data is considered critical for the validation of physics-based, semi-empirical boundarylayer transition prediction methods and to stimulate the validation of laminar numerical chemistry models and the development of turbulence models supporting NASA's next-generation spacecraft under the Constellation program.

The present paper is intended as an introduction to a series of four papers on the viability of obtaining and application of quantitative spatially resolved flight thermography ${ }^{2-5}$. Reference 2 describes key elements associated with mission planning and execution from the perspective of the Navy flight crew that obtained the thermal imagery from onboard a NP-3D Orion aircraft. This reference will describe the aircraft-based optical system known as Cast 
Glance and it's support to the Shuttle program spanning over two decades. A summary of the near infrared (NIR) sensor upgrade that was implemented just prior to the HYTHIRM missions will be provided. Formulation of strategies to develop aircraft test support positions to handle contingencies such as Shuttle one orbit wave-offs/west coast diversions are discussed. Topics such as optimal IR sensor configuration, image acquisition, communications, and lessons learned for future thermal imaging observations are addressed. Reference 3 contrasts the legacy Cast Glance analog IR system to the new digital near infrared (NIR) imaging system (i.e., dynamic range, signal-to-noise) used onboard the Navy Cast Glance aircraft. A description of the calibration strategy and process to characterize this new digital camera is detailed in this reference and focuses on radiometric, spatial and spectral aspects of the NIR imaging system that permitted conversion of captured radiant intensity to temperature values. Comparison of the temperatures inferred from the HYTHIRM imagery to the limited surface thermocouples will be provided to characterize measurement uncertainties. Reference 4 describes the application of a code that was used to generate highfidelity synthetic infrared (IR) signatures to characterize Shuttle radiance during re-entry and how the output from this model was used pre-flight to configure the infrared sensor to optimize dynamic range and mitigate saturation. Image registration, frame averaging and other processes and algorithms used to reduce atmospheric and optical bench blurring effects and improve image quality are discussed. Analytical image registration methods applied post-flight to remove geometric effects of a non-orthogonal projection on the image plane are also described along with techniques used to map the final 2-D temperature images to the actual 3-D Shuttle geometry. Comparisons of surface temperature from the Shuttle surface thermocouples to that inferred from the NIR measurements will be used to validate the methodology and access uncertainties. Finally, in Reference 5, initial comparisons of the NIR derived global surface temperature to numerical laminar and turbulent prediction is presented.

\section{Motivation}

Relative to discrete onboard surface instrumentation, the non-intrusive nature of infrared thermography makes it a very powerful tool to observe surface flow phenomena from a global perspective. Any flow phenomena that create measurable surface temperature changes such as shock wave interactions, flow separation, and boundary layer transition can be visualized. Quantitatively, if surface temperatures associated with a hypersonic laminar and/or fully turbulent boundary layer flow can be inferred from calibrated in flight imagery, they could be used to verify engineering models or numerical predictive methods and associated turbulence models. While most aerospace applications of infrared thermography have been limited to wind tunnel testing, this measurement technique has been utilized during several Shuttle entries over the past 25 years to obtain flight data ${ }^{6-15}$. The renewed interest in thermal imagery during Shuttle entry was initially motivated by the desire to reduce uncertainties associated with an empirical strategy to predict BLT onset. This empirical methodology, adopted to quickly assess thermal environments induced by damage to the Shuttle's TPS, is derived from ground-based measurements ${ }^{16,17}$ that are extrapolated to flight using representative (and limited) flight dat ${ }^{18}$. During the RTF BLT predictive tool development phase, it was recognized that the level of conservatism imposed by these extrapolation uncertainties could be more clearly established and/or reduced with quality data from a controlled roughness flight experiment. Advocacy from the technical community led the Space Shuttle Program (SSP) to support a series of hypersonic boundary layer flight tests. In the initial proposal, an isolated protuberance was to be located on the Shuttle wing to induce boundary layer transition and turbulence at hypersonic conditions ${ }^{19}$. Global temperature IR images with adequate spatial resolution and dynamic range were proposed to non-intrusively complement the discrete thermocouple data on these flight tests by providing spatially continuous surface temperature at targeted Mach number(s).

Applicability of such flight data was not restrictive to Shuttle damage assessment. In general, heating augmentations and temperature increases resulting from hypersonic flight through the atmosphere of Earth (or other planets such as Mars) impose critical requirements on the design of any vehicle thermal protection system (TPS). The onset time and physical location of laminar-to-turbulent transition and subsequent fully turbulent surface heating determine thermal protection system material selection, placement, and thickness (i.e., vehicle design, weight, payload etc.). In terms of vehicle performance, boundary layer transition (BLT) can influence vehicle aerodynamics (i.e., increased drag), landing (or impact) accuracy of autonomously guided spacecraft or delivery systems and scramjet propulsion system aerodynamic performance. The development of numerical tools for the reliable and rapid prediction of BLT, however, continues to be hindered by the inability to rapidly and accurately model the complex physics associated with this processes. Looking towards the future, the present heatshield TPS design philosophy from the Crew Exploration Vehicle (CEV) assumes fully turbulent flow during all phases of entry. However, larger than desired uncertainties in predicted surface temperature from numerical two equation turbulence models have led the technical community to employ more simple algebraic models. If computational techniques are to successfully migrate to the use of two equation turbulence models to predict surface temperatures on complex vehicle shapes 
associated with a fully turbulent boundary layer, flight data will become increasing important in terms of validation of these models. Collectively, a reduction in uncertainties in the prediction of both transition onset and turbulent heating can remove unnecessarily large TPS margins that currently translate to reduced payload capability and degraded mission performance.

\section{Shuttle Entry Infrared Imaging during Return-to-Flight}

A. STS-114 (2005), STS-121 (2006), STS-115 (2006), STS-116 (2006)

In anticipation of a Shuttle flight test program, an effort was made prior to the first return to flight mission (STS114) after the Columbia accident (STS-107) to establish whether or not remote imaging could provide quantitative global surface temperature on the windward surface of the Shuttle during boundary layer transition at high Mach number ${ }^{13,15}$. The ensuing Space Shuttle sponsored effort was leveraged from post STS-107 recommendations made to NASA management to improve imaging capability during ascent and entry. With unprecedented on-orbit photo documentation of damage to the Shuttle TPS system incurred during ascent, the near term goal of the proposed thermal imagery was to assess acreage temperature increases associated with potential damage induced BLT. The aeroheating technical community viewed the SSP sponsored activity as an opportunity to influence the observation objectives and incrementally demonstrate key elements of a quantitative spatially resolved surface temperature measurement capability over a series of flights. As discussed in Refs. 13 and 15, these early missions involved operationally mature assets, but they were flown ad-hoc, that is, with little to no pre-flight mission planning. A specific lack of guidance in optimally configuring the optical systems for initial long-range acquisition and closest approach led to a success rate of under 50\%. The reader is referred to Refs. 13 and 15 for complete details of the four entry-imaging attempts made during STS-114 (July 2005) thru STS-116 (December 2006).

A US Navy aircraft obtained some of the most dramatic imagery from these early observations and in particular, during STS-121 entry. During this mission, on-orbit TPS inspections of Discovery revealed several protruding tile gap fillers. After real time engineering assessment, the gap fillers were not considered a flight safety issue and no spacewalk was performed to remove them. Responding within 72 hours from the initial SSP request, a Navy aircraft was successfully deployed and stationed under the Shuttle ground track near a point in the entry where the Mach number was approximately 12. Near the point of closest approach (Fig.1) the thermal imagery revealed the high temperature footprint of turbulent flow from a protruding gap filler located just upstream of the body flap. Although the area of high heating downstream of the protruding gap filler on STS-121 is clearly evident in this intensity image, quantitative information regarding temperature or the angular spreading of disturbed flow could not be determined because of significant image saturation (white areas). Although promising, the lack of a radiance model precluded any pre-flight sensor simulation to estimate resolution, characterize atmospheric effects, quantify dynamic range and optimize integration times. Never-the-less, the partial success on STS-121 served as the foundation for a proposal to develop a team and the tools necessary to increase the probability of returning scientific engineering quality data on the then proposed Shuttle boundary layer transition flight experiment.

\section{HYTHIRM Project}

Based upon the imagery successes during the Shuttle RTF and recognizing the tremendous technical opportunity that the Shuttle BLT flight tests offered, the NASA Engineering and Safety Center (NESC) sponsored the formation of a team of technical experts to assess existing imaging capability within the US and to develop and validate a mission planning tool set $^{13,20,21}$. The overall goal of the assessment team was to then utilize the validated mission planning tools to determine the viability of obtaining global temperature measurements on a hypersonic flight vehicle using one or more of the nation's

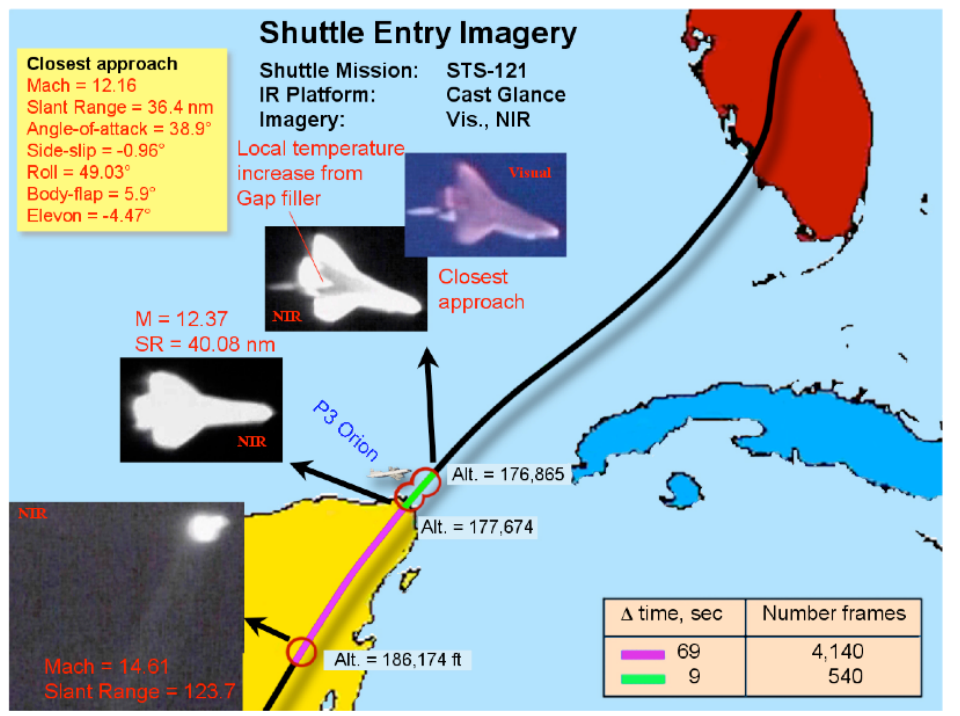

Fig. 1. Cast Glance Entry Imaging in Support of STS-121 
existing suite of applicable imaging assets. The near term target of opportunity to demonstrate capability with available technology was (but not limited to) the Shuttle BLT flight experiment then planned for fall 2008.

\section{A. Optical Assets (2007)}

Under HYTHIRM, an unclassified IR imaging asset database was compiled. While only aircraft were considered pre-HYTHIRM, an understanding of multiple types of imaging platforms was considered as part of a risk reduction strategy. Presently, optical assets in the database are cataloged by their deployment as a land, sea, or air based platform (satellite-based systems have been considered but their potential inclusion into the database has been deferred). Many of these optical systems support specific range operations and as such, only a select few are actually viable in support of Shuttle thermal imaging. However, the database was not compiled exclusively for an assessment against a Shuttle imaging mission. There are a number of other DoD, DARPA, or commercial sector missions on the near term horizon that could potentially benefit from the demonstrated Shuttle thermal imaging capability.

The HYTHIRM database of airborne, land-based and sea-based platforms contains pertinent information on existing capability to rapidly assess optical performance/capability and system mobility. Some of the more relevant system parameters include detector waveband, dynamic range, pixel and array size, instantaneous field of view, integration time, analog or digital format, optical resolution, telescope optical diffraction limits, and aperture/focal length. The information, listed in spreadsheet format, is updated periodically and is readily accessible by the simulation and mission planning tools. The database also includes information on aircraft performance metrics such as ceiling, endurance, cruise speed and range so that consideration of timely asset relocation can be determined. The reader is referred to Ref. 21 for a summary of the asset database.

\section{B. Radiance Modeling and Simulation (2007)}

One of the most significant lessons learned from the Shuttle sponsored entry observations during RTF (20052006) was the lack of mission specific planning tools and corresponding decision making processes and procedures for reliable acquisition and tracking of the Shuttle. That is, the low probability of quantitative imagery obtained under RTF was directly traced to (1) the inability to accurately predict actual sensor response to the infrared signature presented by the Shuttle and (2) the lack of a method to assess and respond to last minute changes to the Shuttle entry trajectory. To address detector response characteristics, HYTHIRM utilized two Shuttle specific radiance models to quantify the Shuttle infrared signature during entry. The first was developed and largely used to highlight general sensor issues and to advocate for hardware modifications to meet imaging requirements ${ }^{22}$. This initial radiance modeling capability was instrumental in underscoring the fact that the legacy analog NIR camera used during the RTF demonstration flights by Cast Glance possessed insufficient dynamic range to completely avoid saturation (as was experienced during STS-121). During actual Shuttle imaging mission operations in 2009, a second higher fidelity radiance model used by DoD to assess plume signatures was adapted for HYTHIRM use. The general modifications and use of this higher fidelity code during a mission is highlighted in a subsequent section and is discussed in more in detail in Ref.4.

The complexity and permutations of Shuttle on-orbit operations makes the accurate determination of the final ground track and Orbiter roll/bank orientation virtually impossible until a few hours before the de-orbit burn is performed. Based upon the RTF experiences, it was essential to develop planning tools that would allow for last minute trajectory updates to be assessed and ensuing recommendations regarding asset placement to be communicated rapidly. A graphics based virtual environment tool was desired to determine the optimum position of imaging asset(s) relative to the Shuttle to ensure mission success. The Virtual Diagnostic Interface (ViDI) ${ }^{23}$ tool developed at LaRC was adapted for HYTHIRM use. In this software package, surface CAD definition of the vehicle targeted for imaging along with 6 degree of freedom trajectory information are imported in a custom ViDI program tied in with commercial off the shelf graphical software to visualize aspects of the entire trajectory on a virtual threedimensional Earth. During a mission, a 3-D Space Shuttle model is animated along the trajectory. The program allows the user to specify an imaging asset (e.g., a particular telescope mount on an aircraft or a land based system) and quickly determine the view/orientation of the shuttle based on the asset position and the location of the Shuttle during entry. 


\section{Validation of Mission Planning Tools (2008)}

A critical element of the systematic HYTHIRM approach was a semi-remote field deployment of optical assets at Sandia National Laboratory whereby radiometric data was collected on a shuttle tile array heated to surface temperature's typical of a Shuttle re-entry. The primary objective of the test ${ }^{20}$ was the demonstration and validation of a suite of mission planning tools including the initial radiance prediction methodology. Testing in the spring of 2008 at the facility located in Albuquerque, NM involved the coordination of infrared imaging assets from five organizations (three land imagers, one airborne and one space-based). The testing complex consists primarily of a 200 foot tall concrete tower known as the Solar Tower located in close proximity to a field of 212 mirrored solar collectors (heliostats) as shown in Fig. 2. Under a more traditional application, the facility is typically used for material response testing of TPS. That is, ablative material TPS samples are

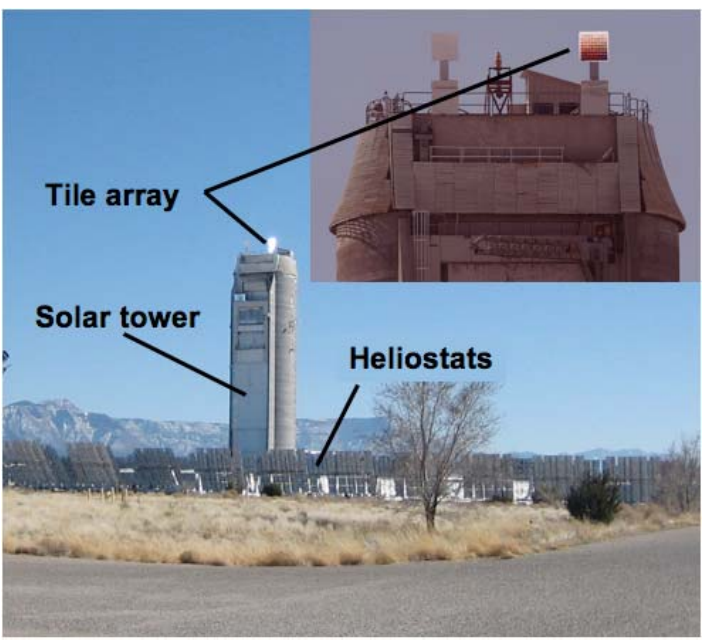

Fig. 2. National Solar Thermal Test Facility mounted to a test stand on top of the tower and exposed to high heat flux levels to assess material response characteristics. In the HYTHIRM sponsored validation test, the facility was utilized in a more unconventional sense. As detailed in Ref 20, a 4 foot by 4 foot test panel, consisting of an 8 by 8 array of LI 900 ceramic tiles, was constructed. These tiles are similar in construction to tiles used on the windward surface of the Shuttle. The top level of the Solar Tower provided an ideal test bed for the HYTHIRM radiometric calibration and validation tests because of the capability of rapidly heating the tile test panel to spatially uniform and non-uniform elevated temperatures in an unsheltered-open-air environment that was conducive to obtaining unobstructed radiometric data by airborne and land-based IR imaging assets. Illumination of the tile array in the visible spectrum can be seen in the inset of Fig. 2. Thermocouples installed on the array and an infrared imager located in close proximity to the test target provided surface temperature measurements. Tests were conducted to obtain radiometric data of a known radiation source in order to characterize potential optical systems for resolved and point source thermal imagery. The over-all test environment, test article and test approach are discussed in detail in Ref. 20.

Results from the Solar Tower tests were used to evaluate the technology readiness of multiple systems and determine their relative priority for deployment in support of future hypersonic flight test programs including the boundary layer transition flight experiment being pursued by the Shuttle program. Of particular relevance was a parametric analysis conducted with the initial radiance mode ${ }^{22}$ which had indicated that the legacy Cast Glance ana$\log$ NIR detector would provide no margin for integration time (exposure) errors or uncertainties associated with actual surface temperatures of the Shuttle during re-entry. The Solar Tower results ${ }^{3}$ indicated that the dynamic range of the analog system used by Cast Glance during the RTF flights was less than 8-bit (256 count) and that if additional Shuttle imaging missions were supported by the Navy aircraft, a 14-bit bit digital NIR camera system would be required to provide increased signal-to-noise and protect against image saturation. The Solar Tower test was also the first opportunity for the HYTHIRM team to collectively perform as a team under conditions associated with a real time remote data collection under somewhat adverse field conditions. By the summer of 2008, the NESC sponsored HYTHIRM project had successfully identified and inventoried relevant optical imaging assets and had developed, matured and validated simulation and modeling tools for assessment and mission planning purposes. The culmination of the imaging assessment task sponsored by the NESC was the application of these tools to identify the best hardware and optical platform configurations and deployment strategies for successful acquisition of quantitative global surface temperature data.

\section{HYTHIRM Application to Shuttle Flights (2008)}

In the fall of 2008, the modeling capability developed under HYTHIRM had characterized the emergence of a point source representation of the Orbiter from the horizon with both day and night sky radiance. As noted in Ref. 22 , this modeling capability was used to identify the probable root cause of image acquisition failure during an adhoc imaging mission associated with a Shuttle daylight re-entry (STS-116). In addition, quantified improvements in procedures associated with tracking, infrared band selection, dynamic range and estimating spatial resolution were made. Postured with a higher probability of future mission success, the HYTHIRM team was tasked by the Shuttle program to provide quantitative thermal imagery to compliment the onboard instrumentation associated with the 
boundary layer transition flight experiment targeted to fly in March 2009. On a basic level, the Shuttle would appear to be an ideal candidate for quantitative thermal imaging. That is, a large vehicle with a well characterized thermal environment re-entering along a pre-determined path. In truth, the reality of supporting an actual Shuttle thermal imaging observation during entry is quite complex with a high risk of failure. For instance, determination of where and when the Shuttle will land is highly uncertain until the end of the mission - at times minutes before the actual de-orbit burn. Actual Shuttle entry de-orbit burn timelines are generally not discussed until the midpoint of each mission when consumable margins are assessed, and the entry weight and de-orbit planning metrics are updated. Under a nominal mission to the International Space Station (ISS), the detailed end-of-mission entry ground track is not issued until the Shuttle undocks with the ISS approximately 2 days before entry. Trajectory predictions done earlier do not account for the detailed conditions of the orbiter (mass, orbit dispersions), real time changes in key mission trajectory events (ISS re-boosts, undock and separation burns, orbit adjust burns to optimize de-orbit opportunities), or a high fidelity atmospheric forecast. To facilitate timely planning, Shuttle ground tracks need to be provided to asset owners as quickly as possible to assess implications of weather or mechanical related waveoffs, Shuttle roll/bank maneuvers, and sun exclusion. Significant planning and re-planning is required to accommodate multiple entry trajectory scenarios. Because of the variability of Shuttle flights returning from the ISS, with landings at both Kennedy space Center (KSC) and Edwards Air Force Base (EDW), asset deployment strategies favor the flexibility and range of airborne systems. In addition, optical systems carried aloft by aircraft minimize atmospheric effects. Air based platforms are not without their own set of logistical challenges. Shuttle ground tracks along with aircraft loiter times and fuel range, determine the allowable Mach coverage for any particular aircraft. Furthermore, it was recognized that high Mach number observation locations (M 18-20) could require that an aircraft fly into foreign airspace.

The HYTHIRM assessment team recommended and the Shuttle program accepted the use of a single operationally mature asset with minimal hardware upgrades to increase the probability of engineering quality data return. Ref. 21 summarizes the trade study and identifies the relevant parameter space from which this deployment recommendation was based.

\section{HYTHIRM Project Support to the BLT Flight Experiments (2009)}

\section{A. Mission Training}

To insure the highest probability of mission success, the HYTHIRM team sought early identification of communication paths to obtain trajectory, meteorological and other information pertinent to mission planning. To facilitate the rapid transfer of information, the HYTHIRM team felt that a presence at NASA Johnson Space Center (JSC) was desirable. This desire led to the formation of a HYTHIRM mission operations team composed of several individuals that operated in proximity to the Shuttle Mission Control Center (MCC). This HYTHIRM team was required to interface with the Shuttle operations team on a noninterference basis. To better understand the information flow and communications processes of the Shuttle Flight Dynamics Officers (FDO) during an actual re-entry, the team was invited to observe the landing phase of several missions prior to the first official HYTHIRM re-entry observation. Based upon these observations, the team developed a HYTHIRM mission operations plan and companion requirements. The team then participated in several follow-on training exercises that consisted of scripted dress rehearsals that were conducted to approximate actual mission operations and contingencies. The dress rehearsals were performed under the direction of a JSC entry flight dynamics officer as shown in Fig. 3. The outcome of these training sessions led to a mission execution plan that defined critical roles, tasks and timelines for the dissemination of critical information during the actual imaging missions.

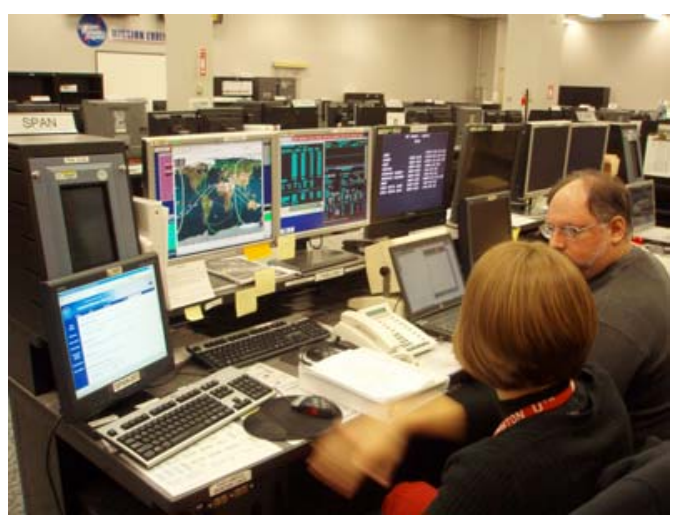

Fig. 3. Mission Dress Rehearsal Positions were identified to support several critical functions such as general mission over-site; the processing of real time Shuttle entry trajectories; communications with air traffic control and any imaging asset(s) using a satellite phone and/or a high frequency radio patch; understanding the implications of the predicted weather at both the landing site and the intended observation location along with actual real-time cloud locations for the determination of a cloud free line of site from the aircraft to the Shuttle. An aerosciences team was responsible for assimilating all the information and recommending the desired Mach number of interest. As part of the aeroscience team, a radiance 
modeling coordinator provided sensor configuration recommendations based upon the anticipated locations of the Shuttle and the imaging aircraft. A JSC FDO liaison to the HYTHIRM team was responsible for interpretation of Shuttle Flight Director and MCC communications, and assisting with the analysis of trajectories.

\section{B. Modeling the Shuttle Infrared Signature}

While the radiance model ${ }^{22}$ (circa 2007) was used successfully to highlight general sensor issues and to advocate for hardware modifications, it was not mature enough to provide synthetic thermal images associated with various sensor configuration settings under the rapid timelines associated with entry. To accelerate the capability of simulating actual detector response, an off the shelf $\operatorname{code}^{24}$ traditionally used by the Department of Defense to support an advanced scene generation capability was used to produce high-fidelity simulated infrared signatures of the Shuttle. This radiance model is fundamentally built around laminar and turbulent CFD surface temperature predictions of the Shuttle over a range of Mach numbers. The conversion to simulated sensor counts takes into account a number of observational, time, and/or environmental-specific variables including: shuttle tile properties (emissivity and reflectivity, Shuttle location, temperature, and view-angle-specific); Shuttle orientation; the Shuttle line of site viewing geometry; atmospheric radiative transfer models including the effects of sun and earthshine; and the sensor model. In the waveband of interest, atmospheric radiance and transmittance is estimated with a widely used radiative transfer code MODTRAN ${ }^{25}$ [MODerate resolution atmospheric TRANsmission], a program designed to model the propagation of electromagnetic radiation through the atmosphere. The reader is referred to Ref. 4 for full details concerning radiance modeling and associated assumptions.

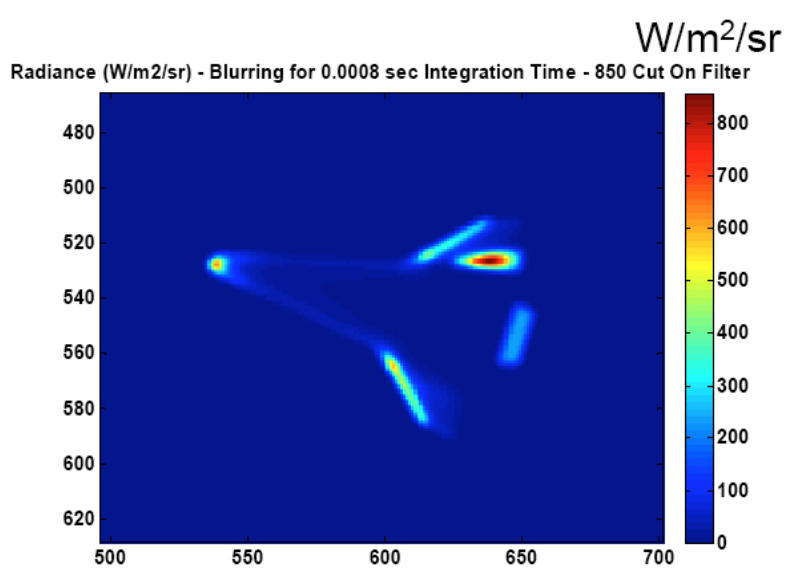

Fig. 4a. Predicted Shuttle Radiance

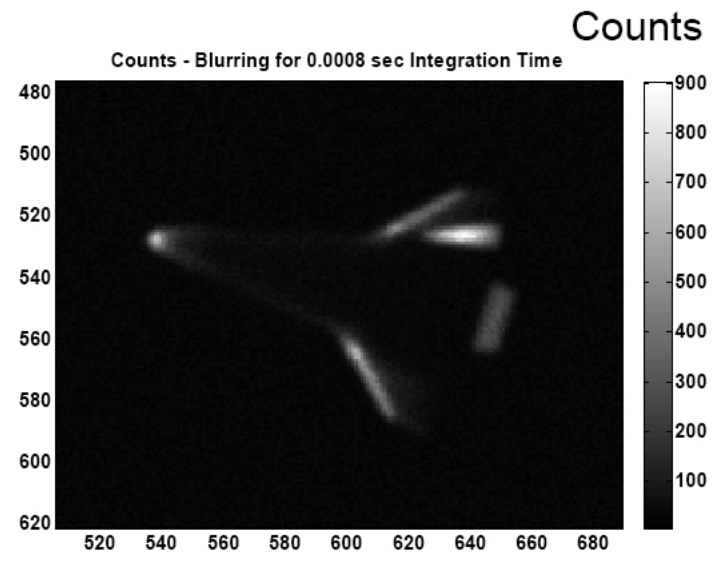

Fig. 4b. Predicted NIR detector Response

The radiance model output is based in a visualization software environment that transforms three dimensional surface temperature input into surface radiant intensity and then projects the three dimensional intensity reaching the sensor onto a two dimensional plane that represents the detector array. The projection can be done for arbitrary orientations of the Orbiter. In terms of output, the radiance model also has the ability to graphically represent the optical systems' expected resolution and image degradation (i.e., blurring) resulting from camera exposure time, atmospheric effects, optical bench motion and/or system optical diffraction limits via a point spread function, if known.

Coupled with the use of operationally mature imaging assets, these simulation tools have allowed the HYTHIRM team to provide sensor operators with pre-flight simulated imagery and recommended detector configurations to optimize the NIR and mid-wave infrared (MWIR) sensor integration times. An example of the radiance modeling output is shown in Fig. 4; the predicted energy reaching the detector in the NIR band for an integration time of $0.008 \mathrm{sec}$ is shown, Fig. $4 \mathrm{a}$, along with the corresponding intensity image simulating the actual detector response, Fig. 4b. A Guassian point spread function was applied to Fig. 4b to represent the effects of anticipated image blurring. This pre-flight knowledge was extremely beneficial to the detector operators who were (in real time) adjusting the integration times manually thus assuring that the raw data would be collected in a manner that would span the sensor's maximum dynamic range, while mitigating against saturation. In contrast to the "ad-hoc" STS-121 imagery shown in Fig 1, this specific mission planning tool was essential in increasing the over-all probability of obtaining scientific/engineering quality imagery during an actual deployment. 


\section{Characterization of NIR Thermal Imager}

As discussed in section IV, results from the Sandi Solar Tower test indicated that the legacy analog NIR system applied to a Shuttle thermal observation during re-entry would possess insufficient dynamic range with poor signal to noise. The HYTHIRM recommendation to the Navy was to replace the analog NIR sensor with an off the shelf digital 12 bit NIR sensor coupled to a laptop based digital acquisition/recording system. The spectral characteristics and features (e.g., manual integration control, recording formats etc.) associated with the Navy's new system are summarized in Refs. 2 and 3.

Prior to the first imaging campaign (STS-119), the new digital NIR camera was characterized by performing radiometric, spatial, and spectral measurements. These characterizations were used as input to the radiance model to predict the imaged NIR radiometric response of the Shuttle and allow for proper selection of the digital camera integration time to minimize pixel saturation. As discussed in more detail in Ref. 3, radiometric characterization was performed by relating measured radiance counts to actual preset temperature values using known blackbody radiation sources. The radiometric calibrations were performed over a range of temperatures of relevance to a Shuttle reentry at specified sensor integration (exposure) times. The resulting calibration curves can then be used to convert measured pixel intensity (counts) to quantitative radiance values. Spot check calibrations were performed during each of the three, STS-119, STS-125, and STS 128, missions. These spot checks involved parking the Cast Glance aircraft at a known distance from the blackbody sources as shown in Fig. 5. The spectral characterization of the Cast Glance optical system used in conjunction with the NIR camera involved the use of a series of long pass filters with known spectral responses. For a given blackbody temperature, the spectral response of the entire imaging system at appropriate frequencies can then be measured thus permitting more accurate temperature measurements. Viewing selected stars during flights while the aircraft was in-flight permitted the spatial characterization of the imaging system. Spatial characterization is important in that such measurements permit the determination of potential motion/vibration-induced blurring. Such advance knowledge allows more accurate pre-flight simulations and the potential to

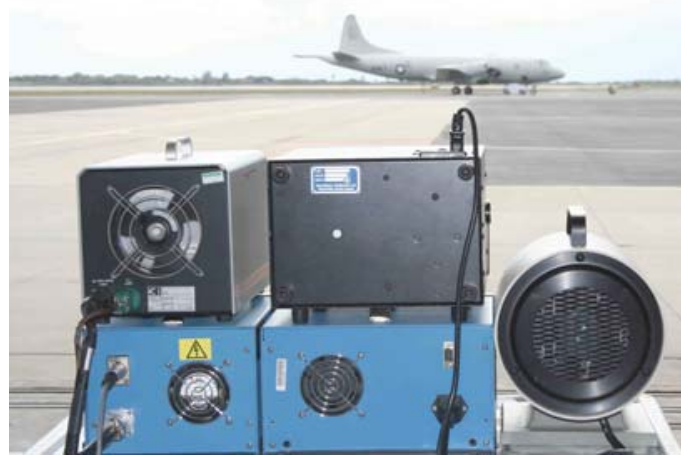

Fig. 5. Calibration Setup using Multiple Blackbody Radiation Sources sharpen blurred images collected during the actual encounter via de-convolution algorithms. Observations of stars prior to or just after data collection the mission is desirable in that local atmospheric conditions along the line of sight can be assessed. In addition, stars of known temperature can provide an additional radiometric assessment to complement the blackbody data. Star observations were generally not possible during the Shuttle missions as all three were performed under daylight conditions. During STS125, a land-based optical system was deployed to the west coast of Florida in an attempt to collect spatially resolved thermal data as the Shuttle approached the KSC. Similar processes were used to characterize this optical system.

\section{Mission Planning and Execution Process: STS-119, STS-125 and STS-128}

Support towards the successful HYTHIRM Shuttle re-entry observations in 2009 has largely been built around lessons learned dating back to the 1981 when the first thermal imaging attempt of the Shuttle with an aircraft ${ }^{11}$ was made during STS-3. Advancements in capability led to additional insight gained during the imaging attempts with a land-based system in $1999^{6,7}$. This methodical approach was continued with incremental experience gained during attempts associated with the 2005-6 ad-hoc missions flown in support of RTF ${ }^{13,15}$. Presently, an optical asset identified for a HYTHIRM mission will spend a significant length of time in preparation using the rigorously developed and validated pre-mission planning tools. For example, the Navy and the crew operating the onboard Cast Glance optical system that supported STS-119, STS-125 and STS-128 observation campaigns typically initiated mission support planning months prior to deployment. As discussed in Ref. 2, this is a step-by-step process that assesses observation requirements against aircraft/Cast Glance system parameters and capability, then casts the recommendations onto the widest dispersion of geographical support scenarios. As indentified earlier, the successful acquisition and tracking of the Space Shuttle during the dynamic hypersonic flight regime requires intricate coordination on many levels.

A successful mission begins with the process of mission planning. A basic review of requirements based upon the desired science is discussed with the asset owner. The desired science determines the specific Mach number that is initially targeted and the orientation of the Shuttle that is desired. Likewise, the science team is educated on the 
limitations of the asset (e.g., range, loiter time, crew duty time, etc.) along with external constraints (e.g., historical adverse weather, uncertain re-entry timelines, prohibited airspace restrictions etc.). The project management team can influence risk posture and mission planning by requiring the aircraft to support multiple entry opportunities per mission day and/or re-deployments to an alternative west coast Shuttle landing site. As the Shuttle mission draws closer, the implications of possible re-entry trajectories and the location of where roll reversals (i.e., left wing up to right wing up) may occur along these preliminary ground track are discussed with the aircraft crew and Cast Glance operators. Understanding roll orientation is essential as it determines orientation of the Shuttle windward surface and hence the asset location relative to the Shuttle ground-track. These collective considerations influence the initial location of where the aircraft is to be located. After the Shuttle is successfully launched, planning and lines of communication between the HYTHIRM mission operations team and the Cast Glance personnel intensifies. When the the Shuttle undocks from the ISS two days prior to re-entry, trajectory updates are issued by the FDO's and sensor configuration recommendations based upon radiance modeling are passed to the Cast Glance personnel. At this point in time, spot check calibrations of the infrared systems are performed on the ground at the staging location. As appropriate, dress rehearsals with the aircraft are conducted to demonstrate and/or verify radio communication paths to the HYTHIRM mission operations team, the Federal Aviation Administration and (if required) any appropriate individual controlling foreign airspace. Re-entry trajectories, roll reversal/Mach numbers locations and de-orbit burn timelines are continually evolving up until the time of ignition for de-orbit burn. For situational awareness, weather conditions at the

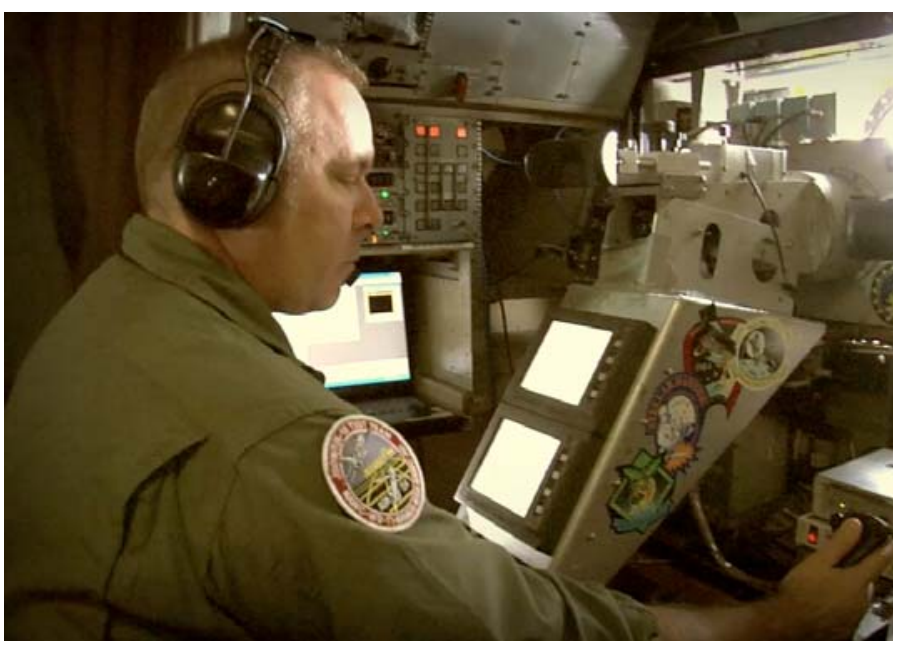

Fig. 6. Cast Glance Operator Configuring the NIR Detector for Optimal Response landing site and in the area of the observation point are continually monitored and updates are provided to the HYTHIRM team by the JSC/NOAA Spaceflight Meteorological Group. Just prior to the Shuttle de-orbit burn, real-time satellite information is processed by the HYTHIRM to identify actual cloud heights and locations. This information is assessed to determine if a more optimal aircraft location was available to provide a cloud free line of site or to mitigate the attenuation of the NIR signature of the Shuttle through high cirrus clouds.

Once on station, pilots and navigators refine the aircraft timing and position, making all necessary adjustments for winds and other local atmospheric conditions. A communications blackout is imposed during the data collection period to permit the aircraft crew and Cast Glance personnel to focus on the mission. During this time, the Cast Glance operators are fine tuning equipment, assessing lighting conditions, making sensor adjustments, recording star calibration data (when applicable), and exercising acquisition simulations as shown in Fig. 6. It is therefore imperative that updated information be rapidly and concisely conveyed to the Cast Glance personnel and the P-3 Orion flight crew just prior to long-range acquisition to effectively adjust for acceptable viewing parameters. If the Shuttle entry is waved-off to the next orbit ( $\sim 1.5$ hours), the communication blackout is lifted to convey the revised information. Naturally, time is vital in terms of re-positioning the aircraft up to 500nm away from the initial observation location. Nominally, the Cast Glance aircraft is stationed at altitude of approximately 25,000 ft during a Shuttle observation. In contrast, the Shuttle is descending thru an altitude of approximately 200,000-180,000 ft depending on the targeted Mach number. At the point of closest approach, the aircraft and it's crew observing the shuttle were approximately 26-41 miles from the Shuttle. Reference 2 provides a more complete synopsis of the mission planning from the perspective of the Cast Glance. The synopsis includes a description of how the Shuttle is initially acquired at long range, tracked through closest approach and details how the aircraft is flown during the crucial few minutes surrounding the imaging encounter.

\section{E. STS-119, STS-125 and STS-128 Quick Look Imagery}

After the Shuttle thermal data collect, a "quick look" analysis is applied to convert the raw intensity counts to a global temperature image within a time period of a few days. The process used to convert the raw image counts to quantitative temperature is described in Ref. 3. The first step in this process involves spatial image stabilization of 
the entire sequence of 2-D images acquired (typically 10,000 - 11,000 individual frames). The stabilized imagery is then reviewed for significant events capture during re-entry such as detection of wake phenomenon (contrail), extent of blurring due to atmosphere and optics vibrations, RCS firings and BLT onset and development. During this analysis phase, the process for selecting the sequence of images with optimal spatial resolution is initiated. The sequence of images providing best resolution is determined by first identifying the point of closest approach between the Shuttle and the aircraft. This is inferred from time synchronized navigational data from both vehicles. Simple averaging techniques are applied to a short sequence of intensity imagery (8 to 16 frames) where the Shuttle orientation, perspective distortions, and optical blurring are minimal. A preliminary calibration curve is then used to convert image pixel counts to a radiance image for the mission specified integration time. The radiance is corrected for atmospheric path transmission, solar scattering from aerosols, and path radiance to produce the in-band radiance at the sensor aperture using MODTRAN atmospheric modeling. The object radiance is converted to temperature by using Planck's black body radiation law and a single in band averaged emissivity value appropriate to RCG tile. In Ref. 3, examples of the 2-D quick look imagery obtained near the point of closest approach for STS-119, STS-125 and STS-128 are presented. In this reference, quick look temperature distributions derived from the HYTHIRM thermal imagery is compared to the Shuttle's onboard thermocouples located along the centerline (hence, simplifying the identification of the thermocouple location along one dimension). As noted in Ref. 3, for temperatures exceeding $1100 \mathrm{deg}$ F, the averaged percent difference between the HYTHIRM and thermocouple derived surface temperatures was $8.4 \%$ (Refs. 1 and 4 quantify the general uncertainties associated with the surface thermocouples and thermal imagery, respectively). The methods for obtaining quick look temperatures are generally acceptable for assessing the initial quality of the imagery but the image processing methods do not include more sophisticated mapping techniques, spectral response functions or surface variations in emissivity to more accurately determine thermocouple location and accuracy.

\section{F. STS-119, STS-125 and STS-128 Detailed Image Analysis}

The quick look imagery products disseminated within days of acquisition quantified mission success and provided a general assessment of image quality. These initial products were intended to complement a long-term analysis strategy. The goal of the high fidelity image analysis was to produce the best quality global flight temperature map and corresponding uncertainty estimates from which advanced numerical computational predictions could be compared against. Similar to the quick look process, the long-term analysis has been focused towards the sequence of data collected near the point of closest approach to provide the maximum spatial resolution possible. As described in Ref. 4, image quality is first improved by accurately registering and then averaging selected intensity image frames, thereby improving signal to noise. The registered and averaged frame is then converted to radiance values using sensor calibration data

Single image frame

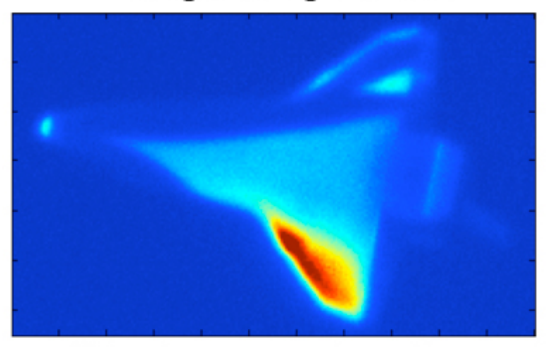

After image registration and averaging of 25 frames
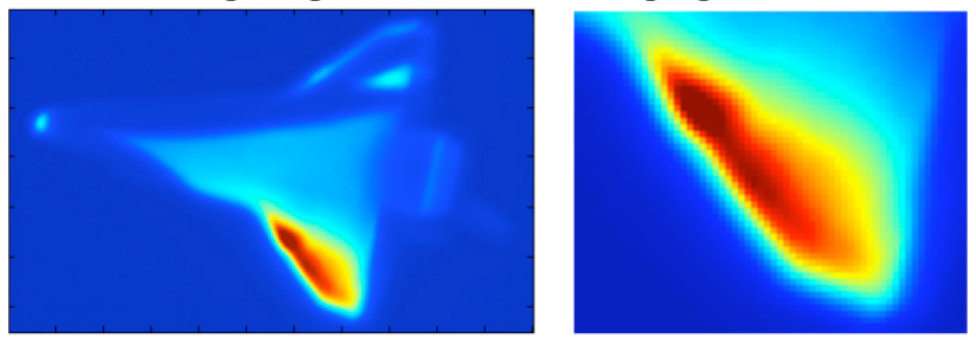

Fig. 7. STS-119 Image Improvement after Image Selection, Registration and Averaging of 25 frames collectively obtained during the three Shuttle observations. Adjustments are applied to account for environmental (i.e., atmospheric) and physical (i.e., surface emissivity etc.) effects. The desired temperature calculation is essentially the reverse of the radiance modeling process used to estimate expected detector performance pre-flight, as previously described. The Shuttle surface temperature at each pixel location is calculated by iterating the Planck blackbody function over the optical filter waveband.

Based upon the sensor and telescope specifications and a target at a nominal range of $38 \mathrm{~nm}$, each pixel associated with the NIR sensor represents approximately 0.84 feet. At this distance, over 100 pixels along the Shuttle length (Full Scale $\sim 122 \mathrm{ft}$ ) are obtained during closest 
approach to the aircraft. However, due to blurring caused by the atmosphere and the optical system, as well as blurring due to jitter and motion, the resolution limit of the acquired unprocessed imagery is estimated to be approximately 3 feet at the $38 \mathrm{~nm}$ range. Because of aircraft motion and vibration, several steps are implemented to minimize the detrimental effects of blurring and jitter. This step-by-step process to improve the image quality is described in Refs. 4 and 26. At a high level, the process entailed the spatial registration of multiple intensity image frames to reduce the detrimental effects from motion (sensor and shuttle), vibration, and atmospherics without compromising the quantitative integrity of the data. Essentially, spatial image registration precisely aligns multiple images so that corresponding image features lie within the same pixel. For the HYTHIRM Shuttle data, multiple image frames were registered using an automated intensity based method to yield a single image frame at the desired condition. After multiple images were registered to a single image, the individual images were then averaged to improve image signal to noise ratio. The results after averaging 25 intensity frames from the STS-119 imagery near the point of closest approach is shown in Fig 7. The top two images in this figure show a single intensity image of the Shuttle and the corresponding zoomed section of the wing leading edge near the location of the expected shockshock interaction. The lower two images in Fig. 7 show the same intensity image after 25 frames were first registered and then averaged. Visual inspections reveal that the pixel variations were reduced with sharper edge definition using the registration and averaging process.

Calibration data are then applied to the improved intensity images to convert intensity to radiance values. These radiance values are then corrected by removing any contributions from the sky background. Transmission losses of this radiance along the line of sight are then estimated by MODTRAN. Satellite weather data and appropriate atmospheric models are used to correct the imagery for absorption and sun illumination effects as the lines of sight changed during re-entry (i.e., during STS-125 the early morning sun directly illuminated the bottom surface of Atlantis in contrast to STS-128 where a late afternoon sun directly illuminated only the top and sides of the Orbiter). As noted by Ref. 4, STS-119 observations were made through a thin cirrus cloud deck, which produced variable foreground irradiance and signal extinction but also generally degraded image resolution.

Once atmospheric corrections were applied, the resulting radiance values were adjusted to a blackbody equivalent value by normalizing with an in-band emissivity appropriate to the Shuttle windward surface RCG tiles at temperature. A uniform emissivity value was applied to the entire radiance image, which is reasonably accurate, except at the nose and wing edges that are comprised of carbon-carbon material. Finally, the corrected/normalized radiance value at each pixel is converted to temperature by iterating the Planck function within the NIR detector wavelength band. That is, initial temperature is given to calculate an initial radiance, and the difference between the pixel radiance and initial estimated radiance is determined. The iteration process of estimating the temperature and computing the radiance continues until the difference with the image pixel radiance is below a specified tolerance. See Ref. 4 for details of the emissivity value and the process described herein.

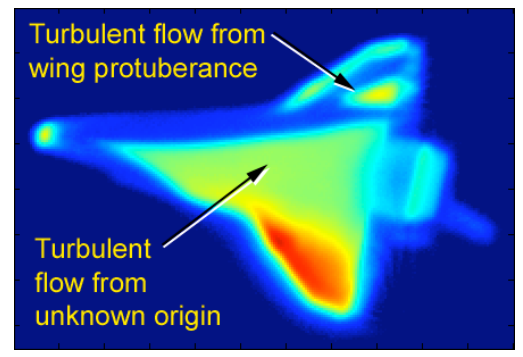

Fig. 8. STS-119 Mach 8.4

Slant Range $\sim 28$ nautical miles Alt. $161 \mathrm{kft}$

Body Flap Deflection $=1.86 \mathrm{deg}$

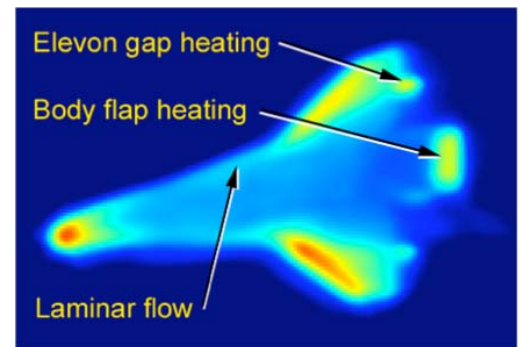

Fig. 9. STS-125 Mach 14.3

Slant Range $\sim 38$ nautical miles Alt. 189 kft

Body Flap Deflection $=1.9 \mathrm{deg}$

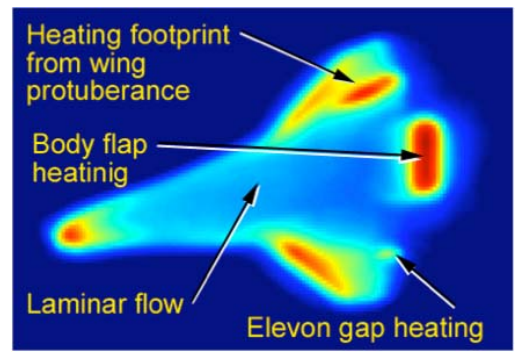

Fig. 10. STS-128 Mach 14.7

Slant Range $\sim 43$ nautical miles Alt. 191 kft

Body Flap Deflection $=7.54 \mathrm{deg}$

\section{G. STS-119, STS-125 and STS-128 Mission Highlights}

The resulting global 2-D surface temperature maps from the STS-119, STS-125 and STS-128 missions derived from the detailed analysis are presented in Figs 8, 9 and 10, respectively. These images correspond to a point near closest approach with the Shuttle orientated for a nearly orthogonal view of the windward surface and maximum spatial resolution. The observations associated with the STS-119 (Fig. 8) and STS-128 (Fig. 10) were associated with Discovery which was configured with a "speed bump" built into a modified tile on the port wing to deliberately disrupt windward surface airflow - and promote transition to turbulence at high Mach numbers ${ }^{1}$. 
The STS-119 Shuttle re-entry in March 2009 was the first HYTHIRM thermal observation mission to benefit from the mission planning tool set. On-orbit TPS inspections of Discovery revealed no significant tile damage or unintentional protruding tile gap fillers. In the absence of any safety of flight issues from TPS damage, the HYTHIRM science team recommended a Mach 8.5 observation point to the Navy aircraft deploying out of KSC. While the BLT onset Mach number was expected near Mach 15, the lower Mach 8.5 deployment balanced constraints associated with Shuttle roll/bank maneuvers during re-entry, foreign airspace restrictions over Cuba and local weather anticipated at the observation site. The weather at the landing site the day of entry was marginal and resulted in a one-orbit wave-off. As discussed by Ref. 2, the HYTHIRM mission operations team at JSC was able to coordinate the relocation of the aircraft to a new test support point within the allocated time. Radio communications with the aircraft permitted the team to provide details of local weather and verbal updates as to the timing and ground track implications of the final de-orbit burn. As anticipated, the pre-flight sensor configuration recommendations provided to Cast Glance resulted in no image saturation and a significant improvement in signal-to-noise relative to the "ad-hoc" flights. Also of significance is that the imagery from the STS-119 mission not only captured the expected thermal footprint of the turbulent flow downstream of the wing protuberance but a much larger area of turbulent flow on the opposing wing that (Fig. 8) that, while not a safety of flight issue, was not anticipated. The surface defect responsible for this asymmetric BLT that occurred at approximately Mach 11.5 was below the detection threshold of the on-orbit inspection imagery and no notable surface discontinuities were observed in the suspected area post-flight. The development of this large area of turbulent flow on the opposing wing was observed long range (over a hundred miles away) with the Shuttle flying at a nominal entry angle of attack of 39 degrees. Thin high altitude cirrus clouds in the line of site were reported by the aircraft crew. Despite these challenges, the sequence of images shown in Fig 11 show what is believed to be the first time that hypersonic transition onset to fully turbulent flow has been observed in flight from global imagery (Shuttle sketch shown for orientation purposes). Figure 11a shows the initial appearance of BLT onset on the starboard wing (lower wing in image) as an increase in intensity brightness (image shown taken at approximately Mach 11.3). Five seconds after this image was taken, the intensity on the starboard wing abruptly drops suggesting a drop in surface temperature associated with flow relaminarization, Fig. 11b. Sixteen seconds after flow re-laminarization, the intensity levels on the wing gradually increased (Fig 11c) indicative of local surface temperature increases as the flow transitioned back to turbulent flow (image taken at approximately Mach 10.6). The intensity variations shown in Fig. 11a-c were initially attributed to possible signal attenuation from high cirrus clouds. However, in-situ thermocouple temperature time histories on the wing corroborated these Mach number specific observations. The phenomenon was also later inferred from time stamped aerodynamic roll and yaw increments (and compensating RCS jet firings) that were consistent with Shuttle asymmetric BLT aerodynamic models derived from previous flight experience. While outside the scope of the initial HYTHIRM objectives, in the future it is possible that additional information can be obtained from such longrange (lower resolution) imagery through the use of advanced de-convolution image processing algorithms.

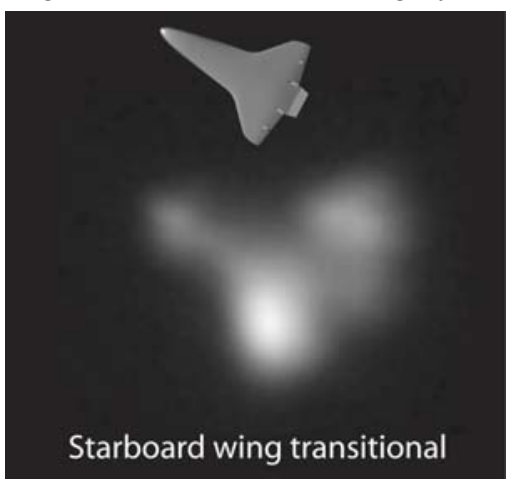

Fig.11a. Long Range NIR Image of Shuttle (STS-119) at Mach 11.3

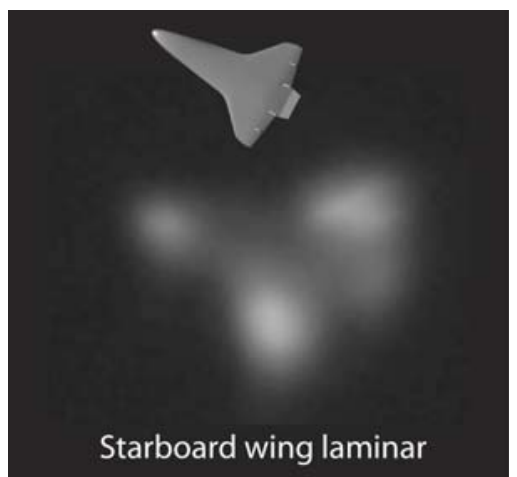

Fig.11b. Long Range NIR Image of Shuttle (STS-119) at Mach 11.1

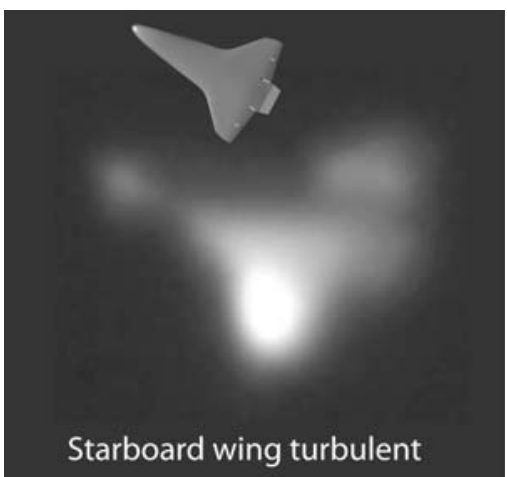

Fig.11c. Long Range NIR Image of Shuttle (STS-119) at Mach 10.6

The remaining two Shuttle observations that HYTHIRM supported in 2009 challenged the team from a logistics perspective. While only landings into KSC were officially supported, the HYTHIRM team actively sought solutions that would permit re-entry imaging if a landing to a west coast diversion site (i.e., White Sands Missile Range or Edwards AFB).

For the STS-125 and STS-128 thermal observations this flexibility proved critical. In contrast to the STS-119 KSC staging base, the Navy aircraft supporting STS-125 and STS-128 was deployed from locations in California and Texas, respectively. These locations permitted the aircraft to support the desired higher Mach observation 
points associated with either a KSC or west coast landing site. As described by Ref. 2, the aircraft transit times from these more centrally located staging areas to the desired Mach 15 observation point still required the crew to begin day of entry mission preparations 8 hours before the actual de-orbit burn. These timelines compelled the mission operations team at JSC to assimilate the most current weather forecast information and render a decision to the Navy to fly east (KSC entry) or west (EDW entry) in advance of the Shuttle entry flight director's decision. In both of these flights, the Shuttle flight director worked towards a KSC entry over a period of several days requiring the Cast Glance aircraft to dispatch to the desired observation locations several times. Ultimately the program diverted the Shuttle to the west coast due to persistent adverse weather at KSC. The HYTHIRM mission operations team was able to anticipate the decision and successfully position the Navy aircraft in the Pacific for nominal Mach 15 thermal observations. As shown in Figs 9 and 10, the thermal images from these two missions flown several months apart are, as expected, very similar. However, close inspection of Figs. 9 and 10 reveal several differences between these flights. Discovery (STS-128), returning with a logistics module from a resupply mission to the ISS, had a center-ofgravity that was shifted 10-inches further aft relative to Atlantis (STS-125). This difference required significantly different flap deflections to maintain the Shuttle at a trimmed condition at 40 deg angle-of-attack. Discovery's (STS-128) body flap was deflected approximately $7.5 \mathrm{deg}(\sim 4$ times that required on STS-125) producing a much higher surface temperature on this control surface. In addition, Discovery was configured with a "speed bump" similar that flown on STS-119 - thus producing an area of elevated heating downstream of the protuberance as observed in Fig. 10. Select temperature profiles and turbulent wedge geometry inferred from the global temperature measurements along with are presented in Ref. 4. Localized heating in the elevon split gap is also apparent in the two Mach 15 thermal images.

\section{H. Supplementary HYTHIRM Observations}

The focus of the HYTHIRM Shuttle analysis has been directed towards the few seconds and the corresponding images centered around the point of closest approach to identify the maximum spatial resolution possible with available technology. However, the actual entire data collection period from initial acquisition of signal to loss of signal often spanned 4-8 minutes with a cumulative total of 10,000 or more individual frames. Initial inspection of this imagery identified several phenomena that may be of interest to specific technical communities in the longer term. At long range, with the Shuttle just above the horizon (not shown) a contrail was evident. As hypothesized by Ref. 4 , the formation of a persistent mesospheric contrail may be produced by either the condensation of the RCS products or ambient gases in the wake. Since the Shuttle's surface is not ablative, ambient mesospheric gases are believed to have been shock-heated, if not ionized, and then to have re-radiated. As the Shuttle passed over the aircraft and receded from view, NIR observations made by the Navy aircraft revealed thermal structure in the wake behind the Shuttle as shown in Fig. 12. Numerous RCS jet firings were clearly evident as shown in Fig. 13. The data set includes wake-RCS plume interaction.

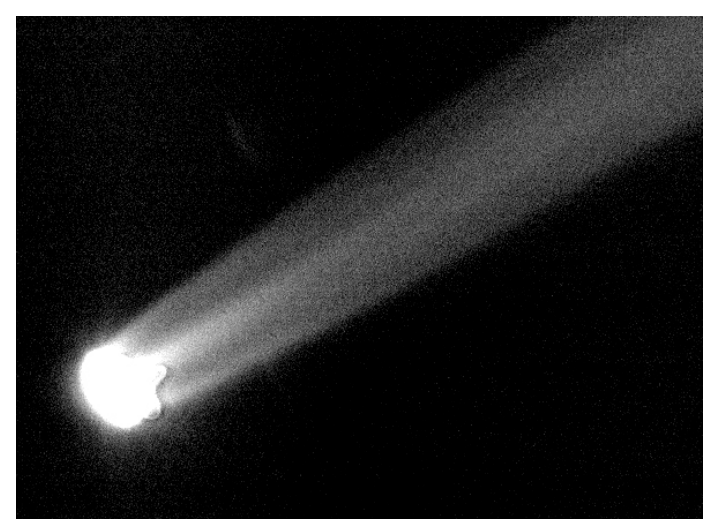

Fig. 12. NIR Image of Shuttle Wake

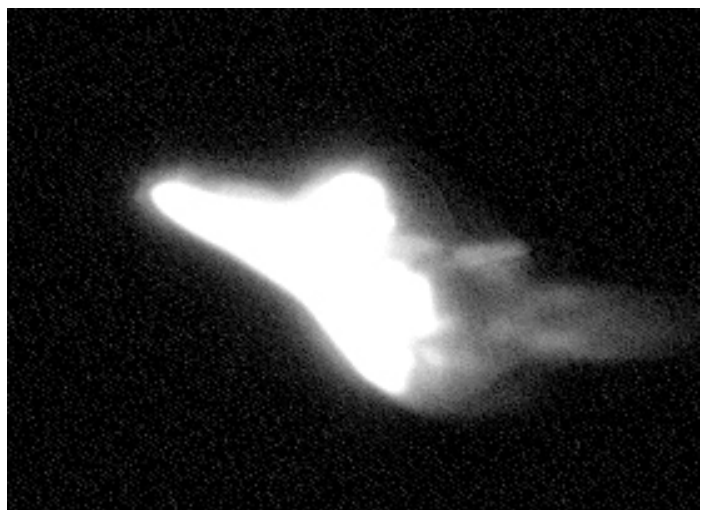

Fig. 13. NIR Image of Shuttle RCS Jet Firing

\section{Comparison to Prediction}

The global HYTHIRM and the companion discrete BLT Flight Experiment measurements have fostered a diverse set of complementary work highlighting a variety of advancements in both experimental technique ${ }^{27,28}$ and numerical modeling ${ }^{29,32-33}$. In terms of CFD, turbulence models play a crucial role in the simulation of complex flows where separation, shock/boundary layer interaction, and flow reattachment are present. The accurate 
prediction of surface temperature associated with nonlaminar flow depends to a large degree on the nature of the turbulence model employed. Surface temperature measurements from the STS-119 boundary-layer transition experiment on Discovery provide a rare opportunity to validate, by assessing accuracy and precision, turbulent CFD models for the prediction of radiative-equilibrium surface temperatures on the windside acreage of the Shuttle at hypersonic reentry conditions. As detailed in Ref. 5, the STS-119 fight data, acquired by on-board thermocouples and the companion global thermal imagery obtained by HYTHIRM team was used for an initial assessment of two specific algebraic turbulence models implemented in the Data Parallel Line-Relaxation (DPLR) ${ }^{34}$ and the Langley Aerothermodynamic Upwind Relaxation Algorithm LAURA $^{35}$ CFD codes along with a more complex twoequation model implemented in DPLR.

In Ref. 5, comparison of temperatures predicted from these turbulence models to STS-119 Shuttle surface thermocouple measurements during re-entry are made to produce quantitative accuracy assessments. Reference 5 provides further details on the methodology and conclusions drawn from these comparisons. In this section, comparison of turbulent CFD prediction and measurement corresponding to the STS-119 HYTHIRM global imagery obtained at a single trajectory point near maximum spatial resolution (Mach 8.4) are highlighted. In order to make accurate comparisons at this condition, the effects of perspective distortion and foreshortening of the image are mitigated by first re-registering the 2-D temperature map (Fig. 8) onto a 3-D Shuttle surface as shown in Fig. 14. The general process of removing geometric effects of nonorthogonal projection on the image plane along with coordinate layover accuracy for the thermocouple locations is described in Ref. 4.

While the HYTHIRM team has largely focused on mission operations to demonstrate a thermal imaging capability, the 3-D mapping capability of Ref. 4 has permitted global HYTHIRM flight measurements be more conveniently ported into graphical post processing tools for comparison to computational prediction. An example of

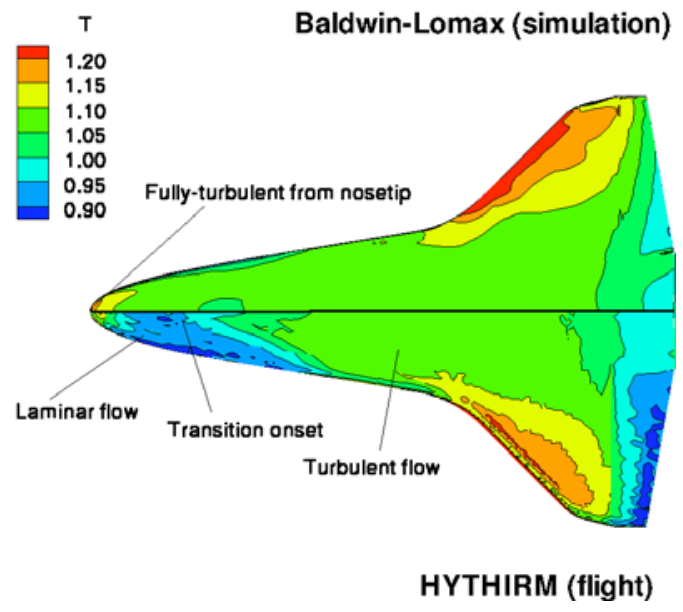

Fig. 15. Comparison of Mach 8.4 Fully Turbulent
CFD with STS-119 Thermal 3-D Mapping

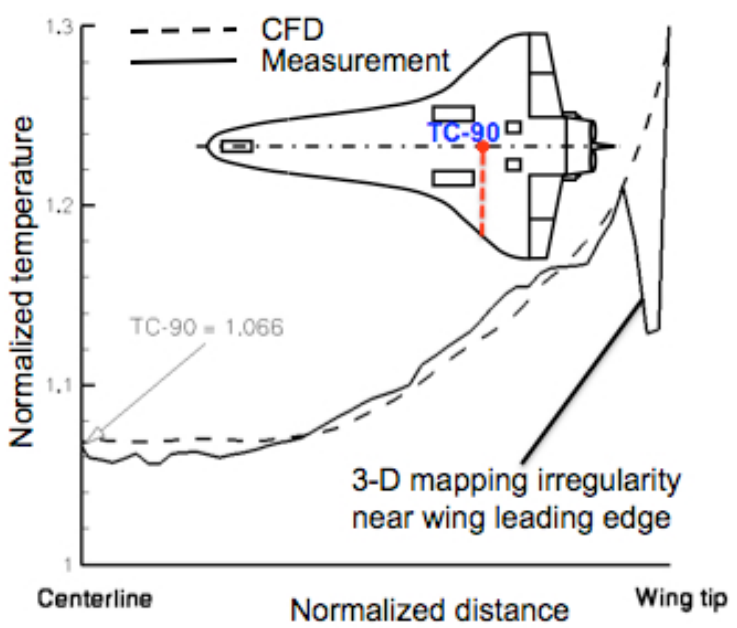

Fig. 16. Comparison of Mach 8.4 Surface Temperature Distribution and Fully Turbulent CFD

the initial comparisons of CFD prediction to HYTHIRM flight measurement taken from Ref 5 (Figs. 15 and 16), suggest the utility and merit of obtaining global flight data. For the first time, numerical predictions can be compared to hypersonic flight measurement in areas of the Shuttle not instrumented with thermocouples. The initial comparison of predicted global surface temperature (upper half) to 3-D mapped STS-119 HYTHIRM flight measurement (lower half) is shown, Fig. 15. The temperatures are expressed in terms of kilo-Kelvins and are displayed 
using a consistent temperature scale. In regions of flow associated with the large asymmetric turbulent wedge on the starboard wing, the corresponding turbulent CFD results are in good agreement in trend and magnitude. In the computations, a fully turbulent boundary layer was assumed from the nose. In contrast, the flight surface temperatures near the nose are associated with a laminar boundary layer (shaded in blue) with transition occurring downstream (general location of onset identified in Fig. 15). In Fig. 16, a turbulent temperature distribution along the starboard wing span was extracted from the global measurement and compared to the turbulent CFD. Both CFD prediction and HYTHIRM derived temperature measurement are within $1 \%$ of the thermocouple located at the centerline. As expected, the algebraic turbulence model at this relatively low hypersonic Mach number (8.4) agrees very well (within 2\%) with the HYTHIRM measurements along the entire wing span with the exception near the wing leading edge where known issues with the current mapping technique exist (see Ref. 4). Comparisons of the acreage thermocouples to HYTHIRM measurement is presented in Ref. 4. Reference 5 presents a more detailed explanation of the computational approach and additional assessments associated with this global comparison.

In the present HYTHIRM image analysis, conversion of intensity images to global temperature was performed via calibration of the NIR detector. To further improve measurement accuracy, temperatures inferred on Reinforced Carbon-Carbon components (nosecap or wing leading edges) could be improved by consideration of the appropriate surface emissivity. In terms of the CFD, improvements in the surface grid could be implemented to take full advantage of the global information. For example, in the present body of work, simplifications to the Shuttle geometry were made such as the omission of the body flap, main engine and orbital maneuvering nozzles. The elevons, while present in the CFD surface grid, were un-deflected and the gap between the inboard and outboard elevon was not modeled.

In Ref. 29, similar types of comparisons to the HYTHIRM global flight data are made. In Ref. 29, a oneequation turbulence model (with compressibility corrections) was utilized along with a novel approach for tripping the boundary layer. In contrast to more traditional methods that specify turbulent onset at a specified circumferential plane along the vehicle axis, the boundary layer was forced turbulent at discrete locations corresponding to the wing protuberance and the estimated location of the disturbance that produced the STS-119 asymmetric boundary layer transition. At the time of this publication, the lateral spreading of the predicted turbulent flow from these discrete (point source) locations displayed good qualitative agreement with that observed in the flight thermal imagery (see Ref. 29).

\section{An Agency and National Vision for Quantitative Imagery}

The successful demonstrations of a remote, non-intrusive quantitative spatially resolved global temperature measurement on the Shuttle with available technologies suggests potential applications towards future hypersonic flight tests within the Air Force, DARPA and NASA as suggested in Fig. 17. HYTHIRM's long term vision supports the application of advanced visual and thermal sensing technology to aid in the development of future aerospace systems and critical technologies to enable highly responsive vehicle operations across the aerospace continuum, spanning reusable space lift and global reach and could be extended to include visual/IR imaging of ascent environments. Such global data facilitates the more accurate assessment of numerical models and engineering methodologies for vehicle design purposes. The HYTHIRM team believes the programs identified below could potentially benefit from quantitative imaging and possibly leverage from the mission planning and operational experiences that evolved from support to Shuttle:

1. DARPA/USAF Falcon ${ }^{36}$ flight test programs supporting a prompt global strike mission objective. The Falcon program will be utilizing assets along the route of flight and in the terminal area to provide radar, optical and thermal imaging for telemetry, tracking and trajectory data. In contrast to Shuttle, a sustained hypersonic cruise capability is required for the prompt global strike mission. Accurate determination of when and where BLT occurs on the vehicle is extremely critical from a TPS and aerodynamic perspective, respectively. For application to this project, the radiation equilibrium wall temperature assumption as an input to the radiance model may not be valid. Alternate material emissivity would need to be quantified for proper image/data reconstruction.

2. $\mathrm{X}-37^{37}$ to quantify test and operational performance envelopes associated with a Shuttle-like winged hypersonic entry vehicle.

3. Constellation ${ }^{38}$ flight testing to quantify environments from post shock and surface radiative heating during Orion re-entry (similar to unresolved Stardust ${ }^{39}$ observations) or ascent convective/plume impingement heating associated with the high altitude launch abort system (LAS). Aerial assets have been used in the past to provide thermal imagery of expendable launchers. Recent imaging support to ARES 1-X has underscored the potential 
to assess/verify pyrotechnic system and aerodynamic performance of future launch architectures such as ARES. After burnout and separation, the ARES first stage will use jets to induce a tumbling motion for a controlled deceleration and a reduced landed footprint for recovery operations. The parachute failure associated with the recovery of the ARES 1-X lower stage highlighted the need for high-resolution imagery. Recognizing the merits of such high resolution visual imagery, the NESC sought HYTHIRM support to provide visual coverage of the Max Abort Launch System (MLAS) demonstration flight out of Wallops. MLAS shroud disposal and parachute deployment sequence was captured at 0.25 -in resolution or better. The CEV Parachute Assembly System Project is presently conducting testing at the Yuma test range to assess parachute deployment characteristics and collect data for validation of CFD for parachute performance. An appropriately designed test using HYTHIRM sponsored planning tools and land-based visual/thermal imagers could provide measurements of the parachute "breathing" process to validate chute performance models. Understanding satellite reentry breakup phenomenology is important to NASA for assessing and mitigating the potential risk to people and property on Earth from surviving space vehicle components. Upper stage breakup and disposal is also important from a range safety perspective and flight imagery could provide data to verify breakup models. NASA has worked with the DoD on several occasions to observe from air-borne platforms the reentry and breakup of spacecraft and launch vehicle stages and document principal breakup altitude, subsequent component explosive events, and debris dispersion characteristics. These airborne observations were at times supplemented with groundbased optical and radar observations. Two recent observations occurred in 1998 and 2000 with the re-entries of Ariane 503 and the Compton Gamma Ray Observatory, respectively. While not sponsored by HYTHIRM an asset owner who supported STS-125 under HYTHIRM direction supported imaging of the European Space Agencies Jules Verne Automated Transfer Vehicle breakup during a guided and controlled scheduled entry over the Pacific Ocean in late 2008. Fragmentation, rotational rates and spectral information were part of the data products.

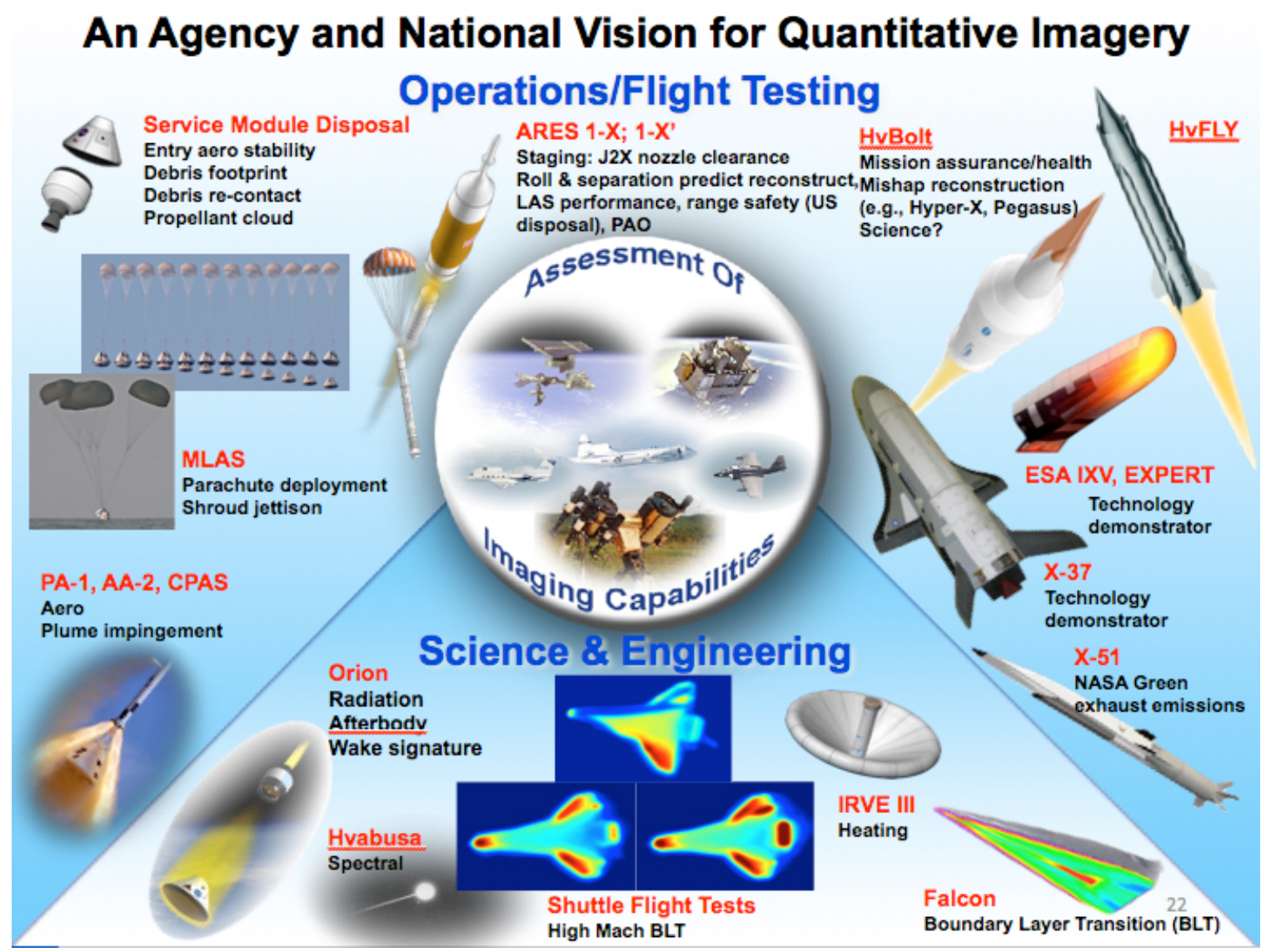

Fig. 17. Programs Potentially Benefiting From a Quantitative Imaging Capability 
4. Vehicle health monitoring could be essential to many flight test programs such as (e.g. US-Australian sounding rocket program HIFiRE, ${ }^{40}$ AFRL/DARPA X-51 Scramjet Engine Demonstrator Waverider Program ${ }^{41}$ or the Army Advanced Hypersonic Weapons program ${ }^{42}$ ). High quality visual and/or thermal imagery could have potentially lead to a more timely closure of previous mishap reconstruction investigations (e.g. Columbia Accident Investigation $^{43}$, Pegasus ${ }^{44}$, Hyper- $^{45}, \mathrm{HYFLY}^{46}, \mathrm{HyBolt}^{47}$ ). The Soyuz anomalies experienced during entry ${ }^{48}$ underscore the risks associated with returning people from ISS despite the use of a historically highly reliable system. The U.S. Air Force is presently re-defining existing imaging requirements for the Evolved Expendable Launch Vehicle (EELV) program. Quality downrange imagery is desired to understand launch anomalies and improve launcher reliability. For example, multi-waveband thermal imaging of the launch sequence has the potential to quantify and characterize the presence of undesirable foreign material in the ascent plume. The desire for such imagery by the commercial launch sector such as Space-X and Orbital has not been assessed.

5. NASA Green Initiatives. With projected increases in aviation growth over the next few decades, environmental issues must be considered from design to implementation. Several new initiatives within the NASA Aeronautics Research Mission directorate will be targeted towards reduced emissions. In partnership with the NASA Science Mission Directorate (SMD) the Aerospace Corporation is presently establishing an organization responsible for conducting studies to better understand the influence of stratospheric emissions from rockets and hypersonic propulsion systems. It has been suggested that thermal imaging associated with hypersonic demonstrator flights such as the AFRL/DARPA X-51 Scramjet Engine Demonstrator Waverider Program ${ }^{49}$ could provide hydrocarbon concentration levels in the wake of the scramjet as inferred from emissions in the NIR spectrum.

6. Space situational awareness. While HYTHIRM did not utilized space assets to support the Shuttle BLT flight tests, a satellite network such as the Space Based Infrared System (SBIRS) ${ }^{50}$ could be used in conjunction with land and air-borne assets to in support of future imaging observations and thus improve the nation's intelligence, surveillance and reconnaissance mission capability.

\section{Summary}

The Hypersonic Thermodynamic Infrared Measurements (HYTHIRM) project is a coordinated and integrated team effort to obtain quantitative, spatially resolved, flight thermography of the Shuttle during hypersonic entry via the development, maturation, validation and application of a suite of mission planning tools. A background and an overview of several multidisciplinary efforts that culminated in the acquisition of high resolution calibrated infrared imagery of the Space Shuttle during hypervelocity atmospheric entry has been presented. Mission planning tools that led to the success of the HYTHIRM team included a pre-flight capability to predict the infrared signature of the Shuttle to simulate detector response characteristics. Such tools provided the rationale for the enhancement and optimization of the hardware/software configuration to increase signal-to-noise and to take advantage of the available dynamic range while mitigating the potential for saturation. The successful collection of thermal data on three Shuttle missions has demonstrated the feasibility of obtaining remote high-resolution infrared imagery during flight for the accurate measurement of surface temperature. To maximize science and engineering return, the acquisition of quantitative thermal imagery during two of the three flights were targeted towards boundary layer transition flight experiments flown on Discovery. In coordination with these two Shuttle flight experiments, a US Navy NP-3D aircraft was flown between 26 to 41 nautical miles below Discovery and remotely monitored surface temperature of the Orbiter at Mach 8.4 (STS-119) and Mach 14.7 (STS-128) using a long-range infrared optical package. This same Navy aircraft successfully monitored the Orbiter Atlantis traveling at approximately Mach 14.3 during its return from the Hubble repair mission (STS-125). Collectively, the spatially resolved global thermal measurements made by HYTHIRM during the Shuttle's hypersonic re-entry have demonstrated the capability to collect scientific quality imagery in a reliable manner using available technology. The calibrated imagery captured by the HYTHIRM team represents the first time the entire surface temperature distribution of a hypersonic vehicle has been captured in flight at these Mach numbers. The thermal imagery obtained during STS-119 re-entry represents the first time that hypersonic transition onset to a fully turbulent flow has been observed globally in flight. In addition to the corresponding surface temperature measurements, the global imagery revealed complex flow field phenomena such as wake/contrail formation and RSC thruster firings that will be of scientific interest to specific technical communities. Post flight, analysis tools were developed and used to assess atmospheric effects and to convert the 2-D intensity images to 3-D temperature maps of the windward surface. Using these global temperature maps, comparison of the flight surface temperature measurements to discrete surface thermocouples and CFD prediction is made and show excellent agreement. The global temperature measurement obtained on the Shuttle using available technologies 
suggests potential future applications (thermal, visual, spectral) towards hypersonic flight test programs within NASA, DoD and DARPA along with flight test opportunities supporting NASA's Constellation program.

\section{Acknowledgments}

The authors would like to acknowledge the fact that without the assistance of the following organizations and individuals the ambitious work performed under the HYTHIRM project would not have been possible. The authors gratefully acknowledge their contributions and behind-the- scenes work:

- Dave Mercer, Ed Shea, Harry Verstynen, Kamran Daryabeigi, Mike Alexander, Scott Berry, Arna Majcher and the rest of the HYTHIRM team, NASA LaRC for perfect mission planning and execution

- Dave Schuster, Christina Cooper, Diana Kerns, Pam Sparks and Pam Throckmorton, NASA Engineering and Safety Center, for technical advocacy and administrative support.

- Robert Barnes, MDA, for technical inspiration after Shuttle RTF

- Robbie Kerns, LaRC Space Operations Program Office for advocacy and support

- Judy Guinn and staff, $45^{\text {th }}$ Space Wing, Cape Canaveral, FL for contractual support

- Ron Dantowitz and Marek Kozubal, MARS Personnel, Clay Center Observatory Dexter Southfield Schools for consultation and support to STS-125

- Mike Hernandez, Kenna Odeghe, Brett Bush and the entire Cast Glance Personnel, Naval Air Warfare Center - Weapons Division, for extreme dedication and ultimately mission execution

- Stephen Tedford, Commanding Officer, and the entire VX-30 "Bloodhounds" squadron and maintenance personnel for getting HYTHIRM to the right spot in the sky

- Dan Hand, CSC/ISTEF for technical support pertaining to calibration and instrumentation hardware/software upgrades

- Chuck Campbell and Brian Anderson, NASA JSC for technical consultation and coordination related to the BLT Flight Experiment

- Jennifer Gruber, Mark McDonald and the Flight Dynamics Group, NASA JSC for providing invaluable mission planning support

- John Shannon and LeRoy Cain, JSC Shuttle Program for advocacy and support

- Tim Oram and the entire Spaceflight Meteorological Group at NASA JSC for their weather forecasting capabilities

- Nicole Lamotte, Olman Carvajal, Peter Jang, and Susan Kwong, Boeing/USA for descent flight trajectories and consultation pertaining to navigation, aerodynamics and the Shuttle instrumentation database

- Martin Ross, Michael Werner and Don Rudy, The Aerospace Corporation for RTF mission planning and initial radiance model development and application

- Don Noah, Terri Murphy, Brenda Eliason and Tracy Calhoun, NASA JSC for insight into the Shuttle onorbit imaging process and advocacy

- Jeff Taylor, Tom Spisz, Mike Kelly, Kwamee Osei-Wusu, Applied Physics Laboratory for technical expertise and image processing; Jim Kouroupis and John Watson, Applied Physics Laboratory for asset identification and technical consultation

- Dan Dexter and the entire CEL imaging lab personnel, NASA JSC for graphical-based mission planning

- Angelo Guastaferro, NIA for project management consultation, leadership and wisdom

- Richard Wheless, NCI Information Systems and Laura Bass, SAIC for assistance in illustration preparation for proposals, briefings and this manuscript

- Bob Blanchard, The George Washington University for re-entry imaging inspiration and technical consultation.

- Jim Hochstetler for calibration logistical support at NAS Corpus Christi, Texas.

- Andrew McCrea, Test Environment Visualization and Support, ATK Space Division, NASA Langley Research Center

- Bill Wood, NASA LaRC for contributions to radiance modeling and CFD support

- Chris Giersch, Blair Allen and the entire NASA EDGE staff for public outreach support

- Kathy Barnstorff and Keith Henry, LaRC Public Affairs; Kyle Herring, JSC Public affairs for media communications and public outreach

- Angel Cases, FAA for coordination of airspace over-flight permissions over Mexico and Central America 


\section{References}

1 Anderson, B., Campbell, C., Kinder, J., Saucedo, L., "Boundary Layer Transition Flight Experiment Overview and In-Situ Measurements," AIAA-2010-240, Jan., 2010.

2 Tack, S., Tomek, D. M., Horvath, T. J., Verstynen, H. A., and Shea, E. J., "Cast Glance Near Infrared Imaging Observations of the Space Shuttle during Hypersonic Re-entry," AIAA Paper 2010-243, Jan. 2010.

3 Zalameda, J. N., Horvath, T. J., Tomek, D. M., Tietjen, A. B., Gibson, D. M., Taylor, J. C., Tack, S., Bush, B. C., Mercer, C. D., and Shea, E. J., "Application of a Near Infrared Imaging System for Thermographic Imaging of the Space Shuttle during Hypersonic Re-entry," AIAA Paper 2010-245, Jan. 2010.

4 Gibson, D. M., Spisz, T. S., Taylor, J. C., Zalameda, J. N., Horvath, T. J., Tomek, D. M., Tietjen, A. B., Tack, S., and Bush, B. C., "HYTHIRM Radiance Modeling and Image Analyses in Support of STS-119, STS-125, and STS-128 Space ShuttleHypersonic Re-entries," AIAA Paper 2010-244, Jan. 2010.

5 Wood, W. A., Kleb, W. L., Tang, C. Y., Palmer, G. E., Hyatt, A. J., Wise, A. J., McCloud, P. L., "Comparison of CFD Predictions with Shuttle Global Flight Thermal Imagery and Discrete Surface Measurements," AIAA Paper 2010-454, Jan. 2010.

6 Blanchard, R.C., Wilmoth, R.G., Glass, C.E., Merski, N.R., Berry, S.A., Bozung, T.J., Tietjen, A., Wendt, J., and Dawson, D., "Infrared Sensing Aeroheating Flight Experiment: STS-96 Flight Results," Journal of Spacecraft and Rockets, Vol. 38, No.4, 2001, pp.465-472.

7 Tietjen, A., Wendt, J., Dawson, D., Ahlgreen, R., Blanchard, R., Welch, S., Lovern, M., "ISAFE Infrared Sensing Aeroheating Flight Experiment" AIAA-BMDO Tech Conf paper 7-9, July 2000.

8 Berry, S.A., Merski, N.R., and Blanchard, R.C., "Wind Tunnel Measurements of Shuttle Orbiter Global Heating with Comparison to Flight," AIAA Paper 2002-4701, August, 2002.

9 Throckmorton, D.A., Zoby, E.V., and Kantsios, A.G., "Shuttle Infrared Leeside Temperature Sensing (SILTS) Experiment," AIAA Paper 85-0328, January, 1985.

${ }^{10}$ Chocol J. C., "Infrared Imagery of Shuttle (IRIS)," Martin Marietta Corporation Final Report, MCR-76-564, Contract NAS29381, August, 1977.

11 "Infrared Imagery of Shuttle (IRIS) Experiment," IRIS/STS-3 Engineering Report, NASA-CR-193052, NASA AMES Research Center, June, 1982.

12 Green, M.J., Budnick, M.P., Yang, L., and Chiasson, M.P., "Supporting Flight Data Analysis for Space Shuttle Orbiter Experiments at NASA Ames Research Center," AIAA Paper 83-1532, June, 1983.

${ }^{13}$ Horvath, T., Berry, S., Splinter, S., Daryabeigi, K., Wood, W., Schwartz, R., and Ross, M., "Assessment and Mission Planning Capability For Quantitative Aerothermodynamic Flight Measurements Using Remote Imaging," AIAA-2008-4022, June 2008.

${ }^{14}$ Berry, S., Horvath, T., Schwartz, R., Ross, M., Campbell, C., Anderson, B., "IR Imaging of Boundary Layer Transition Flight Experiments," AIAA-2008-4026, June 2008.

15 Horvath, T. ., Berry, S. ., Alter, S., Blanchard, R., Schwartz, R., Ross, M., and Tack, S., "Shuttle Entry Imaging Using Infrared Thermography," AIAA-2007-4267, June 2007

${ }^{16}$ Horvath, T. J., Berry, S. A., Merski, N. R., Berger, K. T., Liechty, D. S., Buck, G. M., and Schneider, S. P., "Shuttle Damage/Repair From the Perspective of Hypersonic Boundary Layer Transition - Experimental Results," AIAA-2006-2918, June 2006.

17 Berry, S. A., King, R. A., Kegerise, M. A., Wood, W. A., McGinley, C. B., Berger, K. T., and Anderson, B. A., "Orbiter Boundary Layer Transition Prediction Tool Enhancements,” AIAA-2010-246, Jan., 2010.

18 Berry, S. A., Horvath, T. J., Greene, F. A., Kinder, G. R., and Wang, K.C., "Overview of Boundary Layer Transition Research in Support of Orbiter Return to Flight," AIAA-2006-2918, June 2006.

19 Campbell, C. H., Garske, M. T., Kinder, J., and Berry, S. A., “Orbiter Entry Boundary Layer Flight Testing," AIAA-20080635, Jan., 2008.

${ }^{20}$ Splinter, S., Daryabeigi, K., Horvath, T., Mercer, C.D., Ghanbari, C., Tietjen, A., Schwartz, R., "Solar Tower Experiments for Radiometric Calibration and Validation of Infrared Imaging Assets and Analysis Tools for Entry Aero-Heating Measurements," AIAA-2008-4025, June 2008.

21 Schwartz, R., Ross, M., Baize, R., Horvath, T., Berry, S., Krasa, P., “A System Trade Study of Remote Infrared Imaging for Space Shuttle Re-entry,” AIAA-2008-4023, June 2008.

22 Ross, M., Werner, M., Mazuk, S., Blanchard, R., Horvath, T. ., Berry, S. ., Wood, W., and Schwartz, R., "Infrared Imagery of the Space Shuttle at Hypersonic Entry Conditions," AIAA-2008-0636, 46th AIAA Aerospace Sciences Meeting and Exhibit, Reno, NV, Jan. 7-10, 2008 
23 Schwartz, R. J., McCrea, A. C., "Virtual Diagnostic Interface: Aerospace Experimentation In The Synthetic Environment," MODSIM World Conference and Expo., Oct., 14, 2009.

${ }^{24}$ Crow, D., Coker, C., and Keen, W., "Fast Line-of-Sight Imagery for Target and Exhaust-Plume Signatures (FLITES) Scene Generation Program," Technologies for Synthetic Environments: Hardware-in-the-Loop Testing XI. Edited by Murrer, Robert Lee, Jr.. Proceedings of the SPIE, Volume 6208, June 2006.

25 A. Berk, L.S. Bernstein, D.C. Robertson, “MODTRAN: A moderate resolution model for LOWTRAN7", Report GL-TR-890122, Air Force Geophys. Lab., Bedford, MA, 1989.

${ }^{26}$ Spisz, T. S., Taylor, J. C., Gibson, D. M., Horvath, T. J., Zalameda, J. N., Tomek, D. M., Tietjen, A. B., Tack, S., and Bush, B., "Processing Near-Infrared Imagery of Hypersonic Space Shuttle Reentries," SPIE Paper 7661-17, Apr. 2010, Thermosense XXXII Conference at the SPIE Symposium on Defense, Security, and Sensing.

27 Danehy, P., Ivey, C., Bathel, B., Inman, J., Jones, S., Watkins, A., Goodman, K., McCrea, A., B, Leighty, D., Lipford, W., Jiang, N., Webster, M., Lempert, W., Miller, J. and Meyer, T., "Orbiter BLT flight Experiment Wind Tunnel Simulations: Nearfield Flowfield Imaging and Surface Thermography," AIAA Paper 2010-1571, Jan., 2010.

28 Everhart, J., “Transition Induced by Fence Geometries on Shuttle Orbiter at Mach 10,” AIAA Paper 2010-1577, Jan., 2010.

29 Candler, G. and Campbell, C., "Hypersonic Navier Stokes Comparisons to Orbiter Flight Data," AIAA Paper 2010-455, Jan., 2010 .

30 Yoon, S., Barnhardt, M., and Candler, G. "Simulations of High-Speed Flow over an Isolated Roughness,” AIAA Paper 20101573, Jan., 2010.

31 Choudhari, M., Li, F., Wu, M., Chang, C.-L., Edwards, J.R., Kegerise, M., and King, R.A., "Laminar-Turbulent Transition behind an Isolated Roughness Element in a High-Speed Boundary Layer,” AIAA Paper 2010-1575, Jan., 2010.

32 Tang, C. Y., Trumble, K. A., Campbell, C. H., Lessard, V. R., and Wood, W. A., "Numerical Simulations of he Boundary Layer Transition Flight Experiment," AIAA Paper 2010-453, Jan., 2010.

33 Chang, C., Choudhari, M., and Li, F., "Numerical Computations of Hypersonic Boundary-Layer over Surface Irregularities," AIAA Paper 2010-1572, Jan., 2010.

34 Wright, M. J., Candler, G. V., and Bose, D., "Data-Parallel Line Relaxation Method for the Navier-Stokes Equations," AIAA Journal, Vol. 36, No. 9, Sept. 1998, pp. 1603-1609.

35 Gnoffo, P. A., “An Upwind-Biased, Point-Implicit Relaxation Algorithm for Viscous, Compressible Perfect-Gas Flows," NASA TP 2953, Feb. 1990.

36 Walker, S., Sherk, J., Shell, D., Schena, R., Bergmann, J., and Gladbach, J., “The DARPA/AF Falcon Program: The Hypersonic Technology Vehicle \#2 (HTV-2) Flight Demonstration Phase," AIAA-2008-2539, April-May, 2008.

37 "SpacePlane Flight Reset," Aviation Week \& Space Technology, Sept., 28, 2009.

38 " NASA's Exploration Systems Architecture Study," NASA TM 2005-214062, November 2005.

39 Winter, M, and Herdrich, G., ., "Heat Shield Temperatures and Plasma Radiation Obtained from Spectroscopic Observation of the STARDUST Re-Entry in the Near UV," AIAA-2008-1212, Jan., 2008.

40 Dolvin, D., "Hypersonic International Flight Research and Experimentation (HIFiRE) Fundamental Sciences and Technology Development Strategy", AIAA-2008-2581, April-May, 2008.

41 Borg, M., Schneider, S., and Juliano, T., " Effect of Freestream Noise on Roughness-Induced Transition for the X-51A Forebody," AIAA-2008-592, Jan., 2008.

42 Grossman, E., “Army Eyes Advanced Hypersonic Weapon,” Military.com, Jan., 2007.

43 Columbia Accident Investigation Board Final Report. Vol. I, August, 2003.

44 Covault, C., "Launching Satellites Into Space From an Aircraft Has Special Challenges," Aviation Week \& Space Technology, Aug., 2003.

45 David, L., "X-43A Failure; Source Points to Pegasus Booster," Space.com , June, 2001.

46 Norris, G., "Inquiry Begins Into Hypersonic Missile Failure,” Aviation Week \& Space Technology, Feb., 2008.

47 Berry, S.A., Chen, F., Wilder, M.C., and Reda, D.C., "Boundary Layer Transition Experiments in Support of the Hypersonics Program," AIAA Paper 2007-4266, June. 2007.

48 Morring, F., "Soyuz Ballistic Re-entry Explained," Aviation Week \& Space Technology, May 22, 2008.

49 Borg, M., Schneider, S., and Juliano, T., " Effect of Freestream Noise on Roughness-Induced Transition for the X-51A Forebody," AIAA-2008-592, Jan., 2008.

50 Singer, J., "U.S. Troops Could Get Access to SBIRS High Sensor by August," Space News, May 27, 2008. 